\title{
COUPLED MODE EQUATION MODELING FOR OUT-OF-PLANE GAP SOLITONS IN 2D PHOTONIC CRYSTALS
}

\author{
TOMÁŠ DOHNAL* AND WILLY DÖRFLER*
}

\begin{abstract}
Out-of-plane gap solitons in 2D photonic crystals are optical beams localized in the plane of periodicity of the medium and delocalized in the orthogonal direction, in which they propagate with a nonzero velocity. We study such gap solitons as described by the Kerr nonlinear Maxwell system. Using a model of the nonlinear polarization, which does not generate higher harmonics, we obtain a closed curl-curl problem for the fundamental harmonic of the gap soliton. For gap solitons with frequencies inside spectral gaps and in an asymptotic vicinity of a gap edge we use a slowly varying envelope approximation based on the linear Bloch waves at the edge and slowly varying envelopes. We carry out a systematic derivation of the coupled mode equations (CMEs) which govern the envelopes. This derivation needs to be carried out in Bloch variables. The CMEs are a system of coupled nonlinear stationary Schrödinger equations with an additional cross derivative term. Examples of gap soliton approximations are numerically computed for a photonic crystal with a hexagonal periodicity cell and an annulus material structure in the cell.
\end{abstract}

Key words. gap soliton, photonic crystal, Maxwell's equations, Kerr nonlinearity, out of plane propagation, coupled mode equations, slowly varying envelope approximation, Bloch transformation

AMS subject classifications. 41A60, 35Q61, 35C20, 78M35

1. Introduction. Maxwell's equations for electromagnetic waves in Kerr nonlinear dielectric materials read

$$
\begin{aligned}
\partial_{t} \mathcal{D} & =\nabla \times \mathcal{H}, \\
\mu_{0} \partial_{t} \mathcal{H} & =-\nabla \times \mathcal{E}, \\
\nabla \cdot \mathcal{D} & =0, \\
\nabla \cdot \mathcal{H} & =0
\end{aligned}
$$

for the electric field $\mathcal{E}$, magnetic field $\mathcal{H}$, the electric displacement field $\mathcal{D}$ with the constitutive relations

$$
\begin{aligned}
\mathcal{D} & =\varepsilon_{0}\left(n^{2} \mathcal{E}+\mathcal{P}_{\mathrm{NL}}\right), \\
\mathcal{P}_{\mathrm{NL}, i} & =\sum_{j, l, m=1}^{3} \chi_{i j l m}^{(3)} \mathcal{E}_{j} \mathcal{E}_{l} \mathcal{E}_{m} \quad \text { for } i=1,2,3 .
\end{aligned}
$$

$\varepsilon_{0}, \mu_{0}$ are the electric permittivity and magnetic permeability of vacuum, respectively, $x \mapsto n(x)$ is the refractive index of the medium, and $x \mapsto \chi^{(3)}(x)$ is the cubic electric susceptibility of the medium.

We consider a 2D photonic crystal, i.e. we assume that the material coefficients change periodically on a plane and are independent of the orthogonal component on that plane. Let $a^{(1)}, a^{(2)} \in \mathbb{R}^{3}$ be linearly independent lattice vectors defining the Bravais lattice $\Lambda:=\operatorname{span}_{\mathbb{Z}}\left\{a^{(1)}, a^{(2)}\right\}$ of the crystal. Then the required periodicity reads

$$
\begin{aligned}
n(x) & =n(x+R) \in \mathbb{R}, \\
\chi^{(3)}(x) & =\chi^{(3)}(x+R) \in \mathbb{R}^{3 \times 3 \times 3 \times 3} \quad \text { for all } x \in \mathbb{R}^{3} \text { and all } R \in \Lambda .
\end{aligned}
$$

Without loss of generality we assume that the crystal is homogeneous in the $x_{3}$-direction, i.e. $a_{3}^{(1)}=a_{3}^{(2)}=0$ and $\partial_{x_{3}} n=\partial_{x_{3}} \chi_{i j l m}^{(3)}=0$ for all $i, j, l, m$. We denote by $U$ the Wigner-Seitz cell corresponding to the Bravais lattice. We use $b^{(1)}, b^{(2)}$ to denote the pair of vectors satisfying $a^{(i)} \cdot b^{(j)}=2 \pi \delta_{i, j}$ for $i, j \in\{1,2\}$, and let the reciprocal lattice be $\Lambda^{*}:=\operatorname{span}_{\mathbb{Z}}\left\{b^{(1)}, b^{(2)}\right\}$. $\mathbb{B}$ denotes the first Brillouin zone, i.e. the Wigner-Seitz cell of the reciprocal lattice.

*Institute for Applied and Numerical Mathematics, Karlsruhe Institute of Technology, D-76128 Karlsruhe, Germany 
Note that from the relations in (1.2) it is clear that we are neglecting losses, material dispersion as well as higher order nonlinearities and assuming that the third order nonlinear response of the medium is instantaneous.

We will consider monochromatic waves propagating in the $x_{3}$-direction, i.e. waves propagating out of the plane of periodicity of the $2 \mathrm{D}$ crystal, and use the ansatz

$$
(\mathcal{E}, \mathcal{H}, \mathcal{D})(x, t)=e^{\mathrm{i}\left(\kappa x_{3}-\omega t\right)}(E, H, D)\left(x_{1}, x_{2} ; \omega\right)+\text { c.c. },
$$

where $\kappa \in \mathbb{R}$ and c.c. denotes the complex conjugate of the first term on the right. The ansatz (1.4) contains no higher harmonics, which is valid if the above form of $\mathcal{P}_{\mathrm{NL}}$ is replaced by a time averaged one, see below. Alternatively, a physical justification of neglecting higher harmonics is based on the lack of phase matching and absorption.

Note that for the field (1.4) the divergence free conditions (1.1c) and (1.1d) are automatically satisfied provided $\omega \neq 0$ since the spatially dependent parts

$$
(\hat{\mathcal{E}}, \hat{\mathcal{H}}, \hat{\mathcal{D}})(x ; \omega):=e^{\mathrm{i} \kappa x_{3}}(E, H, D)\left(x_{1}, x_{2} ; \omega\right)
$$

satisfy

$$
\hat{\mathcal{D}}=\frac{\mathrm{i}}{\omega} \nabla \times \hat{\mathcal{H}} \quad \text { and } \quad \mu_{0} \hat{\mathcal{H}}=-\frac{\mathrm{i}}{\omega} \nabla \times \hat{\mathcal{E}}
$$

and thus $\nabla \cdot \hat{\mathcal{D}}=\nabla \cdot \hat{\mathcal{H}}=0$. Since our analysis below is for gap solitons with $\omega$ close to a band edge, the condition $\omega \neq 0$ is for us restrictive only when $\omega=0$ is in a gap and lies near a band edge. Note also that even if higher harmonics are accounted for, the divergence free conditions are still satisfied for $\omega \neq 0$ as (1.5) then holds for each generated harmonic. Clearly, only odd, i.e., $(2 n+1)$-th, $n \in \mathbb{Z}$, harmonics are generated.

We will assume a centrosymmetric and isotropic $\chi^{(3)}$-tensor, which leads to the simplification

$$
\mathcal{P}_{\mathrm{NL}}=\chi_{\mathrm{ci}}^{(3)}(\mathcal{E} \cdot \mathcal{E}) \mathcal{E}
$$

where $\chi_{\mathrm{ci}}^{(3)}:=\chi_{1111}^{(3)}=\chi_{2222}^{(3)}=\chi_{3333}^{(3)}$ for $\chi_{\mathrm{ci}}^{(3)}:\left(x_{1}, x_{2}\right) \in \mathbb{R}^{2} \rightarrow \mathbb{R}$, see [21, Sec. 2d]. Inserting the ansatz (1.4) in the nonlinearity $\mathcal{P}_{\mathrm{NL}}$ clearly generates the harmonics $e^{ \pm 3 i\left(\kappa x_{3}-\omega t\right)}$. These are, however, typically neglected based on the physical arguments that the fundamental harmonics $e^{ \pm \mathrm{i}\left(\kappa x_{3}-\omega t\right)}$ and the higher harmonics are not phase matched and that at the higher values of frequency (i.e. at $\pm 3 \omega$ ) material absorption is usually large preventing the generation of significant fields at these frequencies, see e.g. 9. Considering only the fundamental harmonics, the nonlinear polarization for the ansatz (1.4) becomes

$$
\mathcal{P}_{\mathrm{NL}}=\chi_{\mathrm{ci}}^{(3)}\left(2|E|^{2} E+E \cdot E \bar{E}\right) e^{\mathrm{i}\left(\kappa x_{3}-\omega t\right)}+\text { c.c. },
$$

i.e.

$$
\mathcal{P}_{\mathrm{NL}}=\chi_{\mathrm{ci}}^{(3)}\left(\begin{array}{c}
\left(3\left|E_{1}\right|^{2}+2\left|E_{2}\right|^{2}+2\left|E_{3}\right|^{2}\right) E_{1}+\left(E_{2}^{2}+E_{3}^{2}\right) \bar{E}_{1} \\
\left(2\left|E_{1}\right|^{2}+3\left|E_{2}\right|^{2}+2\left|E_{3}\right|^{2}\right) E_{2}+\left(E_{1}^{2}+E_{3}^{2}\right) \bar{E}_{2} \\
\left(2\left|E_{1}\right|^{2}+2\left|E_{2}\right|^{2}+3\left|E_{3}\right|^{2}\right) E_{3}+\left(E_{1}^{2}+E_{2}^{2}\right) \bar{E}_{3}
\end{array}\right) e^{\mathrm{i}\left(\kappa x_{3}-\omega t\right)}+\text { c.c.. }
$$

Another widely used model for the nonlinear polarization is

$$
\mathcal{P}_{\mathrm{NL}}=\chi_{\mathrm{ci}}^{(3)}[\mathcal{E} \cdot \mathcal{E}]^{\mathrm{av}} \mathcal{E}
$$

where $[f]^{\text {av }}$ denotes the time average of $f$ over the period of $f$, i.e. over $t \in[0, \pi / \omega]$ for $f=\mathcal{E} \cdot \mathcal{E}$, cf. [26, 27]. The averaging generates no higher harmonics so that in this model (1.6) is exact. Note that the Kerr nonlinear problem including all higher harmonics has been recently considered for a 1D periodic structure in [25]. 
In the following we rescale the frequency by defining

$$
\widetilde{\omega}:=\frac{\omega}{c}
$$

but drop the tilde again for better readability. For convenience we will denote the square of the refractive index by

$$
\eta(x):=n^{2}(x) \quad \text { for all } x \in \mathbb{R}^{3} .
$$

With the ansatz (1.4) equations (1.1a) and (1.1b) become

$$
\begin{aligned}
-\mathrm{i} c \omega D & =\nabla \times H+\mathrm{i}\left(\begin{array}{l}
0 \\
0 \\
\kappa
\end{array}\right) \times H, \\
\mathrm{i} c \omega \mu_{0} H & =\nabla \times E+\mathrm{i}\left(\begin{array}{l}
0 \\
0 \\
\kappa
\end{array}\right) \times E .
\end{aligned}
$$

Since all our functions are independent of $x_{3}$, we let from now on $x=\left(x_{1}, x_{2}\right) \in \mathbb{R}^{2}$. Using the fact that $E$ depends only on $x_{1}$ and $x_{2}$, a second order formulation of (1.8) reads

$$
\left(L-\omega^{2} \eta\right) E=\omega^{2} P_{\mathrm{NL}}
$$

where

$$
L E:=\nabla \times \nabla \times E+\mathrm{i} \kappa\left(\begin{array}{c}
\partial_{x_{1}} E_{3} \\
\partial_{x_{2}} E_{3} \\
\partial_{x_{1}} E_{1}+\partial_{x_{2}} E_{2}
\end{array}\right)+\kappa^{2}\left(\begin{array}{c}
E_{1} \\
E_{2} \\
0
\end{array}\right)
$$

and

$$
P_{\mathrm{NL}}=\chi_{\mathrm{ci}}^{(3)}\left(2|E|^{2} E+E \cdot E \bar{E}\right)
$$

Having determined $E$, the magnetic field can be recovered by

$$
H=-\frac{\mathrm{i}}{\omega \mu_{0}}\left(\nabla \times E+\mathrm{i}\left(\begin{array}{l}
0 \\
0 \\
\kappa
\end{array}\right) \times E\right)
$$

Based on the analogy with the periodic nonlinear Schrödinger equation [23], equation (1.9) is expected to have localized $H\left(\operatorname{curl}, \mathbb{R}^{2}\right)$-solutions $E$ for any $\omega$ in a spectral gap of the linear problem $L u=\omega^{2} \eta u$. Such solutions are called gap solitons. The aim of this paper is to provide an approximation of gap solitons $E$ of (1.9) for $\omega$ in an $\varepsilon^{2}$-vicinity $(0<\varepsilon \ll 1)$ of a gap edge using a slowly varying envelope approximation. As we show, envelopes of such gap solitons satisfy a system of nonlinear constant coefficient equations, so called coupled mode equations (CMEs) posed in the slow variables $y=\varepsilon x$. The CMEs can be numerically solved with less effort than the nonlinear Maxwell system (1.9) in the variable $x$. An asymptotic approximation of a gap soliton of (1.9) near a gap edge is then the sum of linear Bloch waves at the edge, modulated by the corresponding envelopes.

Asymptotic approximations via CMEs have been analyzed for gap solitons of the stationary periodic nonlinear Schrödinger equation in 1D [24] as well as in 2D [12, 13, 14]. In these works the approximation via CMEs was also rigorously justified using Lyapunov-Schmidt reductions. Gap solitons of the nonlinear Maxwell's equations have been approximated by CMEs in the case of $1 \mathrm{D}$ photonic crystals with a small (infinitesimal) contrast in the periodicity [16, 24, 25], where [16] considers gap solitons modulated also in time. To our knowledge the problem of a systematic CME approximation of gap solitons of nonlinear Maxwell's equations describing 2D or 3D photonic crystals does not appear in the literature. Although CMEs have been formally derived for pulses in Maxwell's equations with a 2D periodic medium of small contrast 2, 1, 11, these pulses cannot be true gap solitons because in $2 \mathrm{D}$ and $3 \mathrm{D}$ a large enough contrast is necessary for the opening 
of spectral gaps. In this paper we consider a 2D photonic crystal with a finite contrast in the periodicity. For our examples we use a photonic crystal which has several spectral gaps [4].

Besides the above cited works on coupled mode modeling of gap solitons there are a number of papers on the slowly varying envelope approximation of nonlinear pulses in periodic structures with the pulse frequency lying within the spectral bands. The envelope in this case can be typically modeled by the time dependent nonlinear Schrödinger equation and the approximation holds on large but finite time intervals $9,6,6$, 10.

The rest of the paper is organized as follows. In Section 2 we study the linear band structure $\omega_{n}(k)$ of (1.9) (with $\chi_{\mathrm{ci}}^{(3)}=0$ ) and obtain thus the linear spectrum of the problem. We also discuss possible symmetries in the band structure and among the corresponding Bloch waves. An example of a photonic crystal from [4] is then provided, for which the band structure is numerically computed and three band gaps are observed on the positive half axis $\omega>0$. In Section 3 we present a slowly varying envelope approximation of gap solitons of (1.9) for $\omega$ in the vicinity of a spectral edge and carry out a systematic formal derivation of CMEs describing the envelopes. Next, examples of CMEs are presented for the concrete photonic crystal given in Section 2 as well as for other theoretical situations. Here the symmetries in the band structure and among the Bloch waves play an important role in determining properties of the CME coefficients. In Section 4 we plot the approximation of two gap solitons in the chosen photonic crystal. The approximation requires computing the Bloch waves at the edge and solving the corresponding CMEs.

\section{Linear Band Structure.}

2.1. The periodic eigenvalue problem. We study first the linear problem

$$
L u=\omega^{2} \eta u \quad \text { on } \mathbb{R}^{2}
$$

and define the band structure as well as the linear Bloch waves.

By the Bloch-Floquet theory, see [19] or [15, Ch. 3], solution modes of (2.1) are given by the Bloch waves $u_{n}(k ;$.$) for n \in \mathbb{N}$ that satisfy

$$
\begin{aligned}
L u_{n}(k ; .) & =\omega_{n}(k)^{2} \eta u_{n}(k ; .), \\
u_{n}(k ; .+R) & =u_{n}(k ; .) e^{\mathrm{i} k \cdot R} \quad \text { for all } R \in \Lambda,
\end{aligned}
$$

where $k=\left(k_{1}, k_{2}\right)$ sweeps the first Brillouin zone $\mathbb{B} \subset \mathbb{R}^{2}$.

It is well-known that $L$ is self-adjoint and has a compact inverse and that there thus exists a sequence of eigenvalues $\left\{\omega_{n}\right\}_{n \geq 1}$ with $\lim _{n \rightarrow \infty} \omega_{n}=\infty$ and each eigenspace is of finite dimension. These eigenvalues are nonnegative and we use the natural ordering $\omega_{n-1} \leq \omega_{n}$ for $n \geq 1$. The mapping $k \mapsto \omega_{n}(k)$ is called the $n$-th band of the spectral problem (2.2). Of course, (2.2) allows also non-positive bands $-\omega_{n}$. These are typically labeled via $\omega_{-n}=-\omega_{n}$ and will play no role in our analysis. We therefore restrict ourselves to $\omega_{n} \geq 0$ for $n \in \mathbb{N}$. The Bloch waves in (2.2) can be written in the form

$$
u_{n}(k ; x)=p_{n}(k ; x) e^{\mathrm{i} k \cdot x},
$$

where the $p_{n}$ are $\Lambda$-periodic in $x$, i.e. $p_{n}(k ; x+R)=p_{n}(k ; x)$ for all $x \in U, R \in \Lambda$. These satisfy the eigenvalue problem

$$
\begin{aligned}
\left(\widetilde{L}(k)-\omega_{n}^{2}(k) \eta(x)\right) p_{n}(k ; x) & =0 & & \text { for all } x \in U, \\
p_{n}(k ; x+R) & =p_{n}(k ; x) & & \text { for all } x \in \partial U \text { and all } R \in \Lambda,
\end{aligned}
$$

with

$$
\widetilde{L}(k) p_{n}(k ; x)=\left(\nabla+\mathrm{i} k^{\prime}\right) \times\left(\nabla+\mathrm{i} k^{\prime}\right) \times p_{n}(k ; x),
$$


where $k=\left(k_{1}, k_{2}\right) \in \mathbb{B}, k^{\prime}=\left(k_{1}, k_{2}, \kappa\right)^{T}$. Since $p_{n}$ is $x_{3}$-independent, $\widetilde{L}(k)$ can be written as

$$
\widetilde{L}(k)=\left(\begin{array}{ccc}
\kappa^{2}-\left(\partial_{x_{2}}+\mathrm{i} k_{2}\right)^{2} & \left(\partial_{x_{1}}+\mathrm{i} k_{1}\right)\left(\partial_{x_{2}}+\mathrm{i} k_{2}\right) & \mathrm{i} \kappa\left(\partial_{x_{1}}+\mathrm{i} k_{1}\right) \\
\left(\partial_{x_{1}}+\mathrm{i} k_{1}\right)\left(\partial_{x_{2}}+\mathrm{i} k_{2}\right) & \kappa^{2}-\left(\partial_{x_{1}}+\mathrm{i} k_{1}\right)^{2} & \mathrm{i} \kappa\left(\partial_{x_{2}}+\mathrm{i} k_{2}\right) \\
\mathrm{i} \kappa\left(\partial_{x_{1}}+\mathrm{i} k_{1}\right) & \mathrm{i} \kappa\left(\partial_{x_{2}}+\mathrm{i} k_{2}\right) & -\left(\partial_{x_{1}}+\mathrm{i} k_{1}\right)^{2}-\left(\partial_{x_{2}}+\mathrm{i} k_{2}\right)^{2}
\end{array}\right) .
$$

In the variable $k$ the Bloch waves $u_{n}$ and the eigenvalues $\omega_{n}$ are easily proved to fulfill

$$
\omega_{n}(k)=\omega_{n}(k+K), \quad p_{n}(k+K ; x)=p_{n}(k ; x) e^{-\mathrm{i} K \cdot x} \quad \text { for all } x \in U, K \in \Lambda^{*} .
$$

Due to the self-adjoint nature of $\widetilde{L}(k)$ we can normalize the Bloch functions via

$$
\left\langle p_{n}(k ; .), \eta p_{m}(k ; .)\right\rangle=\delta_{n, m},
$$

where $\langle f, g\rangle=\langle f, g\rangle_{L^{2}(U)^{3}}=\int_{U} f(x) \cdot \bar{g}(x) \mathrm{d} x$ for $f, g: \mathbb{R}^{2} \rightarrow \mathbb{C}^{3}$.

For purposes of the later asymptotic analysis of gap solitons we also present calculations of first and second order derivatives of the bands at extremal points. Suppose the band $\omega_{n_{*}}$ has an extremum at $k=k_{*} \in \mathbb{B}$ and denote $\omega_{*}:=\omega_{n_{*}}\left(k_{*}\right)$. By direct differentiation of (2.3) we see that the "generalized Bloch functions" $\partial_{k_{j}} p_{n_{*}}$, for $j \in\{1,2\}$, are solutions of the system

$$
\left(\widetilde{L}\left(k_{*}\right)-\omega_{*}^{2} \eta\right) \partial_{k_{j}} p_{n_{*}}\left(k_{*} ; .\right)=-\partial_{k_{j}} \widetilde{L}\left(k_{*}\right) p_{n_{*}}\left(k_{*} ; .\right) .
$$

Applying the differentiation $\partial_{k_{i}, k_{j}}^{2}$, for $i, j \in\{1,2\}$, to (2.3) and evaluation at $n=n_{*}, k=k_{*}$ yields

$$
\begin{aligned}
\left(\widetilde{L}\left(k_{*}\right)\right. & \left.-\omega_{*}^{2} \eta(x)\right) \partial_{k_{i}, k_{j}}^{2} p_{n_{*}}\left(k_{*} ; x\right) \\
= & 2 \omega_{*} \eta(x) \partial_{k_{i}, k_{j}}^{2} \omega_{n_{*}}\left(k_{*}\right) p_{n_{*}}\left(k_{*} ; x\right)-\partial_{k_{i}, k_{j}}^{2} \widetilde{L}\left(k_{*}\right) p_{n_{*}}\left(k_{*} ; x\right) \\
& -\partial_{k_{i}} \widetilde{L}\left(k_{*}\right) \partial_{k_{j}} p_{n_{*}}\left(k_{*} ; x\right)-\partial_{k_{j}} \widetilde{L}\left(k_{*}\right) \partial_{k_{i}} p_{n_{*}}\left(k_{*} ; x\right) .
\end{aligned}
$$

Necessarily, due to the Fredholm alternative, the right hand side is $L^{2}$-orthogonal to $p_{n_{*}}\left(k_{*} ;\right.$.), which lies in the kernel of $\widetilde{L}\left(k_{*}\right)-\omega_{*}^{2} \eta$ with periodic boundary conditions on $U$. This yields the formula

$$
\begin{aligned}
& \left(\partial_{k}^{2} \omega_{n_{*}}\left(k_{*}\right)\right)_{i, j}=\partial_{k_{i}, k_{j}}^{2} \omega_{n_{*}}\left(k_{*}\right) \\
& \quad=\frac{1}{2 \omega_{*}}\left\langle\partial_{k_{i}, k_{j}}^{2} \widetilde{L}\left(k_{*}\right) p_{n_{*}}\left(k_{*} ; .\right)+\partial_{k_{i}} \widetilde{L}\left(k_{*}\right) \partial_{k_{j}} p_{n_{*}}\left(k_{*} ; \cdot\right)+\partial_{k_{j}} \widetilde{L}\left(k_{*}\right) \partial_{k_{i}} p_{n_{*}}\left(k_{*} ; \cdot\right), p_{n_{*}}\left(k_{*}, \cdot\right)\right\rangle .
\end{aligned}
$$

A straightforward differentiation of $\widetilde{L}(k)$ yields

$$
\begin{aligned}
& \partial_{k_{1}} \widetilde{L}\left(k_{*}\right)=\left(\begin{array}{ccc}
0 & \mathrm{i}\left(\partial_{x_{2}}+\mathrm{i} k_{*, 2}\right) & -\kappa \\
\mathrm{i}\left(\partial_{x_{2}}+\mathrm{i} k_{*, 2}\right) & -2 \mathrm{i}\left(\partial_{x_{1}}+\mathrm{i} k_{*, 1}\right) & 0 \\
-\kappa & 0 & -2 \mathrm{i}\left(\partial_{x_{1}}+\mathrm{i} k_{*, 1}\right)
\end{array}\right) \text {, } \\
& \partial_{k_{2}} \widetilde{L}\left(k_{*}\right)=\left(\begin{array}{ccc}
-2 \mathrm{i}\left(\partial_{x_{2}}+\mathrm{i} k_{*, 2}\right) \mathrm{i}\left(\partial_{x_{1}}+\mathrm{i} k_{*, 1}\right) & 0 \\
\mathrm{i}\left(\partial_{x_{1}}+\mathrm{i} k_{*, 1}\right) & 0 & -\kappa \\
0 & -\kappa & -2 \mathrm{i}\left(\partial_{x_{2}}+\mathrm{i} k_{*, 2}\right)
\end{array}\right), \\
& \partial_{k_{1}}^{2} \widetilde{L} \equiv\left(\begin{array}{lll}
0 & 0 & 0 \\
0 & 2 & 0 \\
0 & 0 & 2
\end{array}\right), \quad \partial_{k_{2}}^{2} \widetilde{L} \equiv\left(\begin{array}{lll}
2 & 0 & 0 \\
0 & 0 & 0 \\
0 & 0 & 2
\end{array}\right), \quad \text { and } \quad \partial_{k_{1}, k_{2}}^{2} \widetilde{L} \equiv\left(\begin{array}{rrr}
0 & -1 & 0 \\
-1 & 0 & 0 \\
0 & 0 & 0
\end{array}\right) \text {, }
\end{aligned}
$$

where $k_{*, j}$, for $j \in\{1,2,3\}$, is the $j$-th component of $k_{*}$. With these the explicit forms of (2.7) read

$$
\begin{aligned}
& \left.\partial_{k_{1}}^{2} \omega_{n_{*}}\left(k_{*}\right)=\frac{1}{\omega_{*}}\left\langle\left(\begin{array}{c}
\mathrm{i}\left(\partial_{x_{2}}+\mathrm{i} k_{*, 2}\right) \partial_{k_{1}} p_{n_{*}, 2}\left(k_{*} ; .\right)-\kappa \partial_{k_{1}} p_{n_{*}, 3}\left(k_{*} ; .\right) \\
\mathrm{i}\left(\partial_{x_{2}}+\mathrm{i} k_{*, 2}\right) \partial_{k_{1}} p_{n_{*}, 1}\left(k_{*} ; .\right)-2 \mathrm{i}\left(\partial_{x_{1}}+\mathrm{i} k_{*, 1}\right) \partial_{k_{1}} p_{n_{*}, 2}\left(k_{*} ; .\right)+p_{n_{*}, 2}\left(k_{*} ; .\right) \\
-2 \mathrm{i}\left(\partial_{x_{1}}+\mathrm{i} k_{*, 1}\right) \partial_{k_{1}} p_{n_{*}, 3}\left(k_{*} ; .\right)-\kappa \partial_{k_{1}} p_{n_{*}, 1}\left(k_{*} ; .\right)+p_{n_{*}, 3}\left(k_{*} ; .\right)
\end{array}\right), p_{n_{*}}\left(k_{*} ; .\right)\right)\right\rangle, \\
& \partial_{k_{2}}^{2} \omega_{n_{*}}\left(k_{*}\right)=\frac{1}{\omega_{*}}\left\langle\left(\begin{array}{c}
-2 \mathrm{i}\left(\partial_{x_{2}}+\mathrm{i} k_{*, 2}\right) \partial_{k_{2}} p_{n_{*}, 1}\left(k_{*} ; .\right)+\mathrm{i}\left(\partial_{x_{1}}+\mathrm{i} k_{*, 1}\right) \partial_{k_{2}} p_{n_{*}, 2}\left(k_{*} ; .\right)+p_{n_{*}, 1}\left(k_{*} ; .\right) \\
\mathrm{i}\left(\partial_{x_{1}}+\mathrm{i} k_{*, 1}\right) \partial_{k_{2}} p_{n_{*}, 1}\left(k_{*} ; .\right)-\kappa \partial_{k_{2}} p_{n_{*}, 3}\left(k_{*} ; .\right) \\
-\kappa \partial_{k_{2}} p_{n_{*}, 2}\left(k_{*} ; .\right)-2 \mathrm{i}\left(\partial_{x_{2}}+\mathrm{i} k_{*, 2}\right) \partial_{k_{2}} p_{n_{*}, 3}\left(k_{*} ; .\right)+p_{n_{*}, 3}\left(k_{*} ; .\right)
\end{array}\right), p_{n_{*}}\left(k_{*} ; .\right)\right\rangle,
\end{aligned}
$$


and

$$
\begin{aligned}
& \partial_{k_{1}, k_{2}}^{2} \omega_{n_{*}}\left(k_{*}\right)=\frac{1}{2 \omega_{*}}\left\langle\left(\begin{array}{c}
-2 \mathrm{i}\left(\partial_{x_{2}}+\mathrm{i} k_{*, 2}\right) \partial_{k_{1}} p_{n_{*}, 1}\left(k_{*} ; \cdot\right)+\mathrm{i}\left(\partial_{x_{1}}+\mathrm{i} k_{*, 1}\right) \partial_{k_{1}} p_{n_{*}, 2}\left(k_{*} ; \cdot\right)+\mathrm{i}\left(\partial_{x_{2}}+\mathrm{i} k_{*, 2}\right) \partial_{k_{2}} p_{n_{*}, 2}\left(k_{*} ; .\right) \\
\left.\mathrm{i}\left(\partial_{x_{1}}+\mathrm{i} k_{*, 1}\right) \partial_{k_{1}} p_{n_{*}, 1}\left(k_{*} ; .\right)+\mathrm{i}\left(\partial_{x_{2}}+\mathrm{i} k_{*, 2}\right) \partial_{k_{2}} p_{n_{*}, 1}\left(k_{*}, \cdot\right)-2 \mathrm{i}\left(\partial_{x_{1}}+\mathrm{i} k_{*, 1}\right) \partial_{k_{2}} p_{n_{*}, 2}\left(k_{*} ; .\right)\right) \\
-2 \mathrm{i}\left(\partial_{x_{2}}+\mathrm{i} k_{*, 2}\right) \partial_{k_{1}} p_{n_{*}, 3}\left(k_{*} ; .\right)-2 \mathrm{i}\left(\partial_{x_{1}}+\mathrm{i} k_{*, 1}\right) \partial_{k_{2}} p_{n_{*}, 3}\left(k_{*} ; .\right)
\end{array}\right)\right. \\
& \left.+\left(\begin{array}{c}
-\kappa \partial_{k_{2}} p_{n_{*}, 3}\left(k_{*} ; .\right)-p_{n_{*}, 2}\left(k_{*} ; .\right) \\
-\kappa \partial_{k_{1}} p_{n_{*}, 3}\left(k_{*} ; .\right)-p_{n_{*}, 1}\left(k_{*} ; .\right) \\
-\kappa\left(\partial_{k_{1}} p_{n_{*}, 2}\left(k_{*} ; .\right)+\partial_{k_{2}} p_{n_{*}, 1}\left(k_{*} ; .\right)\right)
\end{array}\right), p_{n_{*}}\left(k_{*} ; .\right)\right)^{2} .
\end{aligned}
$$

2.2. Symmetries of the Band Structure and the Bloch waves. Symmetries in the refractive index function $\eta$ yield symmetries in the band structure and among Bloch waves. We restrict our attention to the cases of discrete rotational and axial reflection symmetry, which are relevant for the example we present below. The results of this section will be important when determining properties of the coefficients of coupled mode equations in Section 3.4

2.2.1. Rotational symmetry. Assume that the photonic crystal satisfies the rotational symmetry

$$
\eta(x)=\eta\left(r_{\alpha}(x)\right) \quad \text { for all } x \in \mathbb{R}^{2}
$$

for some $\alpha \in(-\pi, \pi]$ with the rotation $r_{\alpha}$ defined by

$$
r_{\alpha}(x)=\left(\begin{array}{c}
\cos (\alpha) x_{1}-\sin (\alpha) x_{2} \\
\sin (\alpha) x_{1}+\cos (\alpha) x_{2}
\end{array}\right)
$$

Below we use the notation $r_{\alpha}(v)=\left(\cos (\alpha) v_{1}-\sin (\alpha) v_{2}, \sin (\alpha) v_{1}+\cos (\alpha) v_{2}\right)^{T}$ if $v$ is a two dimensional vector $v \in \mathbb{C}^{2}$ and $r_{\alpha}(v)=\left(\cos (\alpha) v_{1}-\sin (\alpha) v_{2}, \sin (\alpha) v_{1}+\cos (\alpha) v_{2}, v_{3}\right)^{T}$ if $v$ is a three dimensional vector $v \in \mathbb{C}^{3}$.

The symmetry (2.12) implies a symmetry of the Rayleigh quotient corresponding to the eigenvalue problem (2.3) and thus a symmetry of the band structure. In detail, for $k \in \mathbb{B}$ we have

$$
\omega_{n}^{2}(k)=\min _{\substack{V \subset H_{\text {per }}^{\text {cer }} \\ \operatorname{dim} V=n}} \max _{w \in V, w \neq 0} \frac{\int_{U}\left|\left(\nabla+\mathrm{i} k^{\prime}\right) \times w(x)\right|^{2} \mathrm{~d} x}{\int_{U} \eta(x)|w(x)|^{2} \mathrm{~d} x},
$$

and the corresponding extremal point is $p_{n}(k ;$.$) . Due to the relation$

$$
\left(\left(\nabla+\mathrm{i} r_{\alpha}\left(k^{\prime}\right)\right) \times f\right)\left(r_{\alpha}(x)\right)=r_{\alpha}\left[\left(\nabla+\mathrm{i} k^{\prime}\right) \times r_{-\alpha}\left(f\left(r_{\alpha}(x)\right)\right)\right] \quad \text { for all smooth } f: \mathbb{R}^{2} \rightarrow \mathbb{R}^{3}
$$

we get

$$
\int_{U}\left|\left(\nabla+\mathrm{i} r_{\alpha}\left(k^{\prime}\right)\right) \times w(x)\right|^{2} \mathrm{~d} x=\int_{U}\left|\left(\nabla+\mathrm{i} k^{\prime}\right) \times r_{-\alpha}\left(w\left(r_{\alpha}(x)\right)\right)\right|^{2} \mathrm{~d} x,
$$

and symmetry (2.12) yields

$$
\int_{U} \eta(x)|w(x)|^{2} \mathrm{~d} x=\int_{U} \eta(x)\left|r_{-\alpha}\left(w\left(r_{\alpha}(x)\right)\right)\right|^{2} \mathrm{~d} x .
$$

As a result we obtain that

$$
\omega_{n}(k)=\omega_{n}\left(r_{\alpha}(k)\right) \quad \text { for all } n \in \mathbb{N} \text { and all } k \in \mathbb{B} .
$$

If $\omega_{n}(k)$ has geometric multiplicity one as an eigenvalue of (2.3), we have also a symmetry of the corresponding Bloch functions, namely

$$
p_{n}\left(r_{\alpha}(k) ; x\right)=e^{\mathrm{i} a} r_{-\alpha}\left(p_{n}\left(k ; r_{\alpha}(x)\right)\right) \quad \text { for all } n \in \mathbb{N} \text { and some } a=a(n) \in \mathbb{R} .
$$


Note that a renormalization of $p_{n}\left(r_{\alpha}(k) ; x\right)$, in order to obtain $a=0$ in (2.14), is in general impossible when $r_{\alpha}(k) \doteq k$, where $k \doteq l$ reads " $k$ congruent to $l$ " and means $k=l+K$ for some $K \in \Lambda^{*}$. This is because in this case $p_{n}\left(r_{\alpha}(k) ; x\right)$ and $p_{n}(k ; x)$ are related by (2.4) and a renormalization of the left hand side of (2.14) would affect the right hand side in the same way. When $r_{\alpha}(k)$ is not congruent to $k$, e.g. when $k \in \operatorname{int}(\mathbb{B}) \backslash\{0\}$, then one can set $a=0$ in (2.14).

¿From the symmetry (2.13) we can deduce a symmetry of the second derivatives of $\omega_{n}$. Using the identity $\partial_{k} \omega_{n}(k)=\partial_{k}\left(\omega_{n}\left(r_{\alpha}(k)\right)\right)=\left(r_{\alpha}\right)^{T}\left(\partial_{k} \omega_{n}\right)\left(r_{\alpha}(k)\right)$, we get by further differentiation

$$
\left(\begin{array}{c}
\partial_{k_{1}}^{2} \omega_{n}\left(r_{\alpha}(k)\right) \\
\partial_{k_{2}}^{2} \omega_{n}\left(r_{\alpha}(k)\right) \\
\partial_{k_{1}, k_{2}}^{2} \omega_{n}\left(r_{\alpha}(k)\right)
\end{array}\right)=\left(\begin{array}{ccc}
\cos ^{2}(\alpha) & \sin ^{2}(\alpha) & -\sin (2 \alpha) \\
\sin ^{2}(\alpha) & \cos ^{2}(\alpha) & \sin (2 \alpha) \\
\frac{1}{2} \sin (2 \alpha) & -\frac{1}{2} \sin (2 \alpha) & \cos (2 \alpha)
\end{array}\right)\left(\begin{array}{c}
\partial_{k_{1}}^{2} \omega_{n}(k) \\
\partial_{k_{2}}^{2} \omega_{n}(k) \\
\partial_{k_{1}, k_{2}}^{2} \omega_{n}(k)
\end{array}\right)
$$

for all $k \in \mathbb{B}$ and $n \in \mathbb{N}$.

2.2.2. Reflection symmetry. If the photonic crystal satisfies the reflection symmetry

$$
\eta(x)=\eta\left(S_{1}(x)\right) \quad \text { for all } x \in \mathbb{R}^{2}, \text { where } S_{1}(x)=\left(-x_{1}, x_{2}\right)^{T},
$$

then similarly to Section 2.2 .1 we have

$$
\omega_{n}(k)=\omega_{n}\left(-k_{1}, k_{2}\right) \quad \text { for all } k \in \mathbb{B} \text { and } n \in \mathbb{N} .
$$

Again, if $\omega_{n}(k)$ has geometric multiplicity one as an eigenvalue of (2.3), then

$$
p_{n}\left(S_{1}(k) ; x\right)=e^{\mathrm{i} a} S_{1}\left(p_{n}\left(k ; S_{1}(x)\right)\right) \quad \text { for all } n \in \mathbb{N} \text { and some } a=a(n) \in \mathbb{R},
$$

where $S_{1}(v)=\left(-v_{1}, v_{2}, v_{3}\right)^{T}$ for $v \in \mathbb{C}^{3}$. Just as above, unless $k \doteq S_{1}(k)$, we can set $a=0$ in (2.18). The symmetry (2.17) implies

$$
\begin{aligned}
& \partial_{k_{1}}^{2} \omega_{n}(k)=\left(\partial_{k_{1}}^{2} \omega_{n}\right)\left(-k_{1}, k_{2}\right), \quad \partial_{k_{2}}^{2} \omega_{n}(k)=\left(\partial_{k_{2}}^{2} \omega_{n}\right)\left(-k_{1}, k_{2}\right), \\
& \partial_{k_{1}, k_{2}}^{2} \omega_{n}(k)=-\left(\partial_{k_{1}, k_{2}}^{2} \omega_{n}\right)\left(-k_{1}, k_{2}\right)
\end{aligned}
$$

for all $k \in \mathbb{B}$ and $n \in \mathbb{N}$.

An analogous discussion, of course, applies for the reflection symmetry $\eta(x)=\eta\left(S_{2}(x)\right)$ for all $x \in \mathbb{R}^{2}$, where $S_{2}(x)=\left(x_{1},-x_{2}\right)^{T}$. One the obtains

$$
\begin{aligned}
& \partial_{k_{1}}^{2} \omega_{n}(k)=\left(\partial_{k_{1}}^{2} \omega_{n}\right)\left(k_{1},-k_{2}\right), \quad \partial_{k_{2}}^{2} \omega_{n}(k)=\left(\partial_{k_{2}}^{2} \omega_{n}\right)\left(k_{1},-k_{2}\right), \\
& \partial_{k_{1}, k_{2}}^{2} \omega_{n}(k)=-\left(\partial_{k_{1}, k_{2}}^{2} \omega_{n}\right)\left(k_{1},-k_{2}\right)
\end{aligned}
$$

for all $k \in \mathbb{B}$ and $n \in \mathbb{N}$ and if $\omega_{n}(k)$ has geometric multiplicity one as an eigenvalue of (2.3), then

$$
p_{n}\left(S_{2}(k) ; x\right)=e^{\mathrm{i} a} S_{2}\left(p_{n}\left(k ; S_{2}(x)\right)\right) \quad \text { for all } n \in \mathbb{N} \text { and some } a=a(n) \in \mathbb{R} .
$$

2.2.3. Combination of rotational and reflection symmetries. If both the reflection symmetry (2.16) and the rotational symmetry (2.12) for some $\alpha \in(-\pi,-\pi],|\alpha| \neq \pi / 2$, hold, then for $k$ along the rays with angles $\pi / 2-\alpha / 2$ and $-(\pi / 2+\alpha / 2)$ the mixed derivative $\partial_{k_{1}, k_{2}}^{2} \omega_{n}(k)$ can be expressed in terms of $\partial_{k_{1}}^{2} \omega_{n}(k)$ and $\partial_{k_{2}}^{2} \omega_{n}(k)$. This is because for $k$ along these rays we have $\left(-k_{1}, k_{2}\right)=r_{\alpha}(k)$ or $\left(k_{1},-k_{2}\right)=r_{\alpha}(k)$, so that both (2.15) and (2.19) or (2.20) apply. In detail, suppose

$$
\left(-k_{1}, k_{2}\right)=r_{\alpha}(k) \text {, i.e. } k=|k| e^{\mathrm{i}(\pi / 2-\alpha / 2)} \quad \text { or } \quad k=|k| e^{-\mathrm{i}(\pi / 2+\alpha / 2)}=-|k| e^{\mathrm{i}(\pi / 2-\alpha / 2)} .
$$

Then it follows that

$$
\partial_{k_{1}}^{2} \omega_{n}(k)=\left(\partial_{k_{1}}^{2} \omega_{n}\right)\left(-k_{1}, k_{2}\right)=\cos ^{2}(\alpha) \partial_{k_{1}}^{2} \omega_{n}(k)-\sin (2 \alpha) \partial_{k_{1}, k_{2}}^{2} \omega_{n}(k)+\sin ^{2}(\alpha) \partial_{k_{2}}^{2} \omega_{n}(k),
$$


where the first equality holds due to (2.19) and the second due to (2.15). As a result, for $\alpha \in(-\pi, \pi],|\alpha| \neq \pi / 2$, and $k= \pm|k| e^{\mathrm{i}(\pi / 2-\alpha / 2)}$ we get

$$
\partial_{k_{1}, k_{2}}^{2} \omega_{n}(k)=\frac{1}{2} \tan (\alpha)\left(\partial_{k_{2}}^{2} \omega_{n}(k)-\partial_{k_{1}}^{2} \omega_{n}(k)\right) .
$$

Identity (2.22) applies also in the case when the $S_{2}$ reflection symmetry and the rotational symmetry (2.12) are both present for some $\alpha \in(-\pi,-\pi],|\alpha| \neq \pi / 2$. Then (2.22) holds for $k$ that satisfy

$$
\left(k_{1},-k_{2}\right)=r_{\alpha}(k) \text {, i.e. } k= \pm|k| e^{-\mathrm{i} \alpha / 2} .
$$

2.3. Example: Hexagonal Lattice with a Circular Material Structure. As an example we consider the hexagonal lattice in the $\left(x_{1}, x_{2}\right)$-plane generated by the vectors

$$
a^{(1)}=a_{0}\left(\begin{array}{c}
\cos (\pi / 3) \\
\sin (\pi / 3)
\end{array}\right) \quad \text { and } \quad a^{(2)}=a_{0}\left(\begin{array}{l}
1 \\
0
\end{array}\right) \quad \text { with } \quad a_{0}>0
$$

In the Wigner-Seitz cell $U$ the material structure is given by the annulus centered at the lattice point in the origin and having outer and inner radii $a_{0} / 2$ and $a_{0}(1.31 / 4.9)$ respectively. The material properties are given by $\eta(x)=2.1025$ for $a_{0}(1.31 / 4.9) \leq|x| \leq a_{0} / 2$ and $\eta(x)=1$ otherwise. This is the same as the crystal used in [4, where the corresponding band structure was also computed. One choice of vectors generating the reciprocal lattice is

$$
b^{(1)}=\frac{2 \pi}{J_{12}}\left(\begin{array}{c}
a_{2}^{(2)} \\
-a_{1}^{(2)}
\end{array}\right)=\frac{2 \pi}{a_{0} \sin (\pi / 3)}\left(\begin{array}{l}
0 \\
1
\end{array}\right), \quad b^{(2)}=\frac{2 \pi}{J_{12}}\left(\begin{array}{c}
-a_{2}^{(1)} \\
a_{1}^{(1)}
\end{array}\right)=\frac{2 \pi}{a_{0}}\left(\begin{array}{c}
1 \\
-\cot (\pi / 3)
\end{array}\right),
$$

where $J_{12}=\operatorname{det}\left(a^{(1)}, a^{(2)}\right)=a_{1}^{(1)} a_{2}^{(2)}-a_{2}^{(1)} a_{1}^{(2)}$. These vectors have been obtained via the formulas $\tilde{b}^{(1)}=$ $2 \pi \frac{\tilde{a}^{(2)} \times \tilde{\tilde{a}}^{(3)}}{J_{12}}$ and $\tilde{b}^{(2)}=2 \pi \frac{\tilde{a}^{(3)} \times \tilde{a}^{(1)}}{J_{12}}$, where $\tilde{a}^{(j)}=\left(a^{(j)^{T}}, 0\right)^{T}, \tilde{b}^{(j)}=\left(b^{(j)^{T}}, 0\right)^{T}$ for $j \in\{1,2\}$ and $\tilde{a}^{(3)}=(0,0,1)^{T}$, cf. [5, Ch. 5]. Figure 2.1 shows the crystal geometry and the corresponding Brillouin zone.

In this case both the rotational symmetry (2.12) with $\alpha=\pi / 3$, the reflection symmetry (2.16) as well as the analogous symmetry $S_{2}$ do hold. The band structure and Bloch waves can therefore be recovered via (2.13), (2.14) and (2.17), (2.18) from the irreducible Brillouin zone $\mathbb{B}_{0}$ in Figure 2.1, i.e. the triangle with vertices $\Gamma, M, K$, where $\Gamma=(0,0)^{T}, M=\frac{1}{2} b^{(2)}$, and $K=\frac{1}{\sqrt{3}}\left|b^{(2)}\right|(1,0)^{T}$. These points are called high symmetry points.

Next we provide some specific information about the values of the second derivatives of $\omega_{n}$ at the high symmetry points of the Brillouin zone at hand using symmetries (2.15), (2.19), and (2.20). This information will be used in Section 3.4

Identity (2.15) with $k=0$ and $\alpha=\pi / 3$ yields

$$
\partial_{k_{2}}^{2} \omega_{n}(\Gamma)=\partial_{k_{1}}^{2} \omega_{n}(\Gamma) \quad \text { and } \quad \partial_{k_{1}, k_{2}}^{2} \omega_{n}(\Gamma)=0 \quad \text { for all } n \in \mathbb{N}
$$

Symmetry (2.20) implies

$$
\partial_{k_{1}, k_{2}}^{2} \omega_{n}(K)=0 \quad \text { for all } n \in \mathbb{N} .
$$

At $k=r_{2 \pi / 3}(M)\left(=\frac{1}{2} b^{(1)}\right)$ we have $k_{1}=0$ so that (2.19) implies

$$
\partial_{k_{1}, k_{2}}^{2} \omega_{n}\left(r_{2 \pi / 3}(M)\right)=0 \quad \text { for all } n \in \mathbb{N} .
$$

Relation (2.22) then yields

$$
\partial_{k_{2}}^{2} \omega_{n}\left(r_{2 \pi / 3}(M)\right)=\partial_{k_{1}}^{2} \omega_{n}\left(r_{2 \pi / 3}(M)\right) \quad \text { for all } n \in \mathbb{N} .
$$


(a)

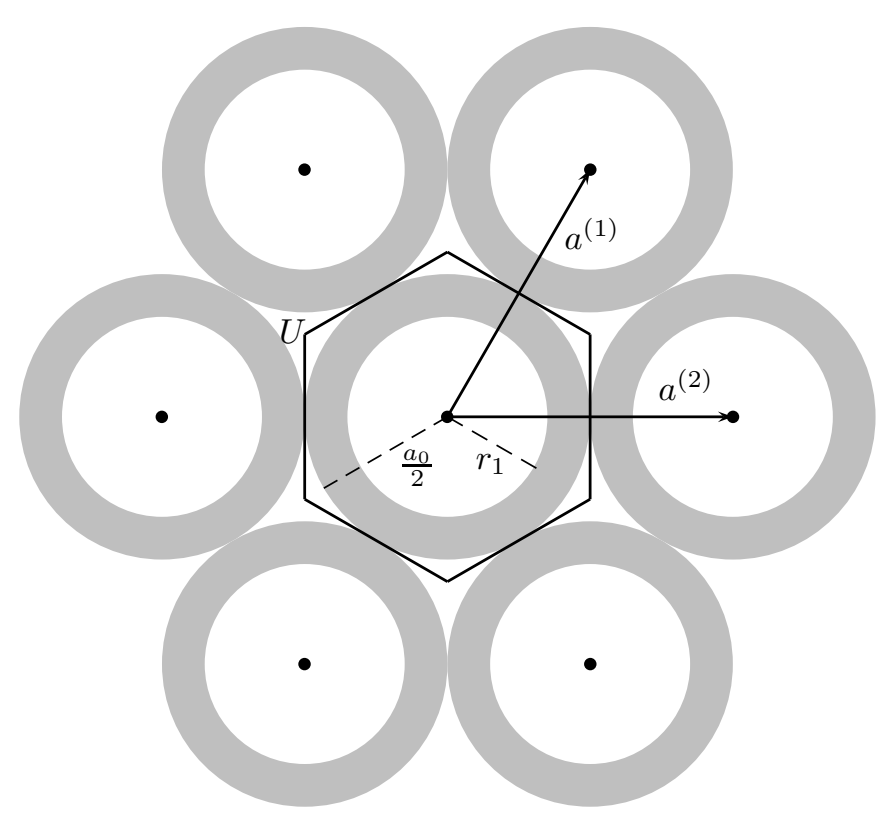

(b)

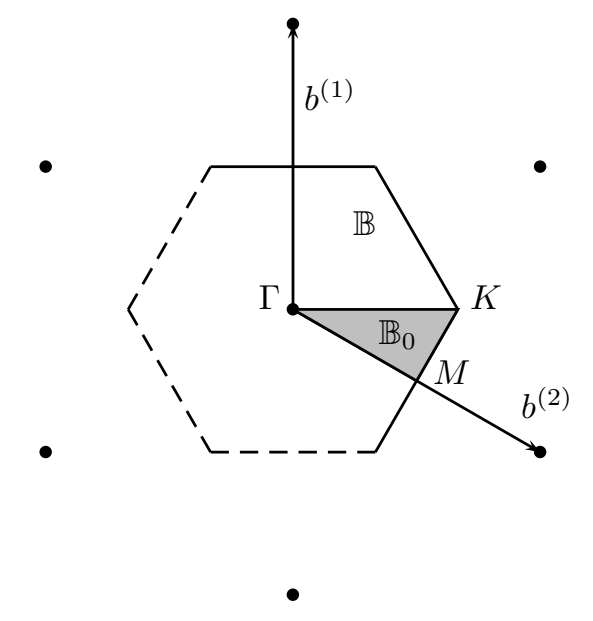

Fig. 2.1: (a) Hexagonal lattice with a cylindrical material structure, (b) the corresponding first Brillouin zone $\mathbb{B}$ with a shaded irreducible Brillouin zone $\mathbb{B}_{0}$. Note that the Brillouin zone has been scaled to fit the figure.

Applying now (2.15) with $\alpha=2 \pi / 3$, we get

$$
\partial_{k_{1}}^{2} \omega_{n}(M)=\partial_{k_{1}}^{2} \omega_{n}\left(r_{2 \pi / 3}(M)\right), \partial_{k_{2}}^{2} \omega_{n}(M)=\partial_{k_{1}}^{2} \omega_{n}\left(r_{2 \pi / 3}(M)\right), \text { and } \partial_{k_{1}, k_{2}}^{2} \omega_{n}(M)=0
$$

for all $n \in \mathbb{N}$. Because $r_{\pi / 3}(M)$ is obtained from $r_{2 \pi / 3}(M)$ by the reflection $\left(k_{1}, k_{2}\right) \rightarrow\left(k_{1},-k_{2}\right)$, we also have

$$
\partial_{k_{1}}^{2} \omega_{n}(M)=\partial_{k_{1}}^{2} \omega_{n}\left(r_{\pi / 3}(M)\right), \partial_{k_{2}}^{2} \omega_{n}(M)=\partial_{k_{1}}^{2} \omega_{n}\left(r_{\pi / 3}(M)\right), \text { and } \partial_{k_{1}, k_{2}}^{2} \omega_{n}\left(r_{\pi / 3}(M)\right)=0 .
$$

As an example we took the configuration from [4] as described in Section 2.3. The computations were done with a finite element Maxwell solver that uses lowest order Nedelec elements [22]. These elements were implemented in the software deal.II [7]. The eigenvalue problems were solved by a Krylov-Schur method *

We computed the eigenvalues $\left\{\omega_{n}(k)\right\}_{n=1,14}$ and corresponding eigenfunctions $\left\{p_{n}(k, \cdot)\right\}_{n=1,14}$ for each vertex $k$ in a discretization of the Brillouin zone $\mathbb{B}$. The error level of this computations is about $10^{-3}$ in the curl-norm and it is estimated from a series of computations on a sequence of nested grids.

In Figure 2.2 we present the numerically computed band structure over $\partial \mathbb{B}_{0}$ (following the tradition) for the above described crystal and for $\kappa=5\left(2 \pi / a_{0}\right)$. Here, $\partial \mathbb{B}_{0}$ is represented by $128 k$-points. It has, however, been checked that the observed gaps do not get narrower in the interior of $\mathbb{B}$. Three band gaps appear on the positive half of the $\omega$ axis, one between 0 and $\omega_{1}$, another one between $\omega_{6}$ and $\omega_{7}$ and the last one between $\omega_{12}$ and $\omega_{13}$.

To get the extremal points at the band edges we used a bisection method in $k$ which was initialised with data obtained from the band structure computation. The approximations to 1st and 2 nd order derivatives of

${ }^{*}$ SLEPc package (HTTP://WwW.GRYCAP.UPV.ES/SLEPC/) 
$k \mapsto \omega_{n}(k)$ at the extremal values were obtained by first projecting $k \mapsto \omega_{n}(k)$ onto a locally quadratic finite element space and then taking mean values of the derivatives around vertices.

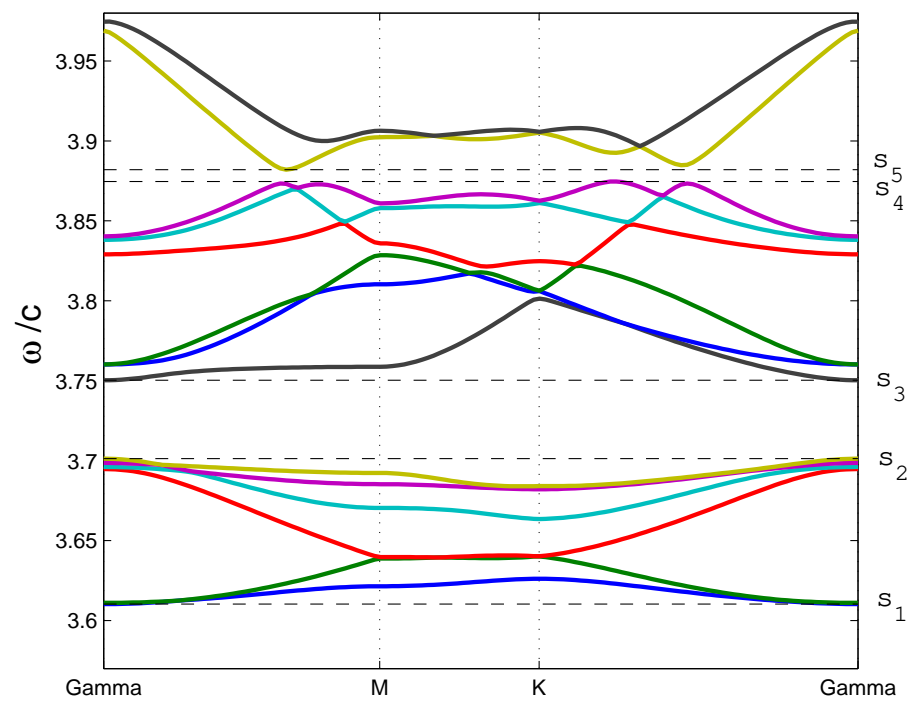

Fig. 2.2: Band structure $k \mapsto \omega_{n}(k)$ for the described hexagonal lattice with the cylindrical material structure: the first 14 eigenvalues along $\partial \mathbb{B}_{0}$. Three band gaps appear on the positive half axis $\Omega \geq 0$ : one between 0 and $\omega_{1}$, one between $\omega_{6}$ and $\omega_{7}$, and one between $\omega_{12}$ and $\omega_{13}$. Gap edges are marked by $s_{1}, \ldots, s_{5}$.

\section{Derivation of Coupled Mode Equations for Gap Solitons near Band Edges.}

3.1. Slowly varying envelope approach. We seek gap solitons $E$ of (1.9). Afterward, the full electric field can be recovered via (1.4).

In the following let us assume that

(A1) the spectrum $\left\{\omega_{n}(k): k \in \mathbb{B}, n \in \mathbb{N}\right\}$ possesses a gap,

(A2) one of the gap edges, denoted by $\omega_{*}$, is attained at precisely $N \in \mathbb{N}$ points $k^{(1)}, \ldots, k^{(N)} \in \mathbb{B}$ by bands with indices $n_{1}, \ldots, n_{N}$, respectively, where the $k$-points and/or band indices are not necessarily all distinct,

(A3) for each $j \in\{1, \ldots, N\}$ the band $\omega_{n_{j}}$ is twice continuously differentiable in $k$ at $k=k^{(j)}$,

(A4) $\partial_{k}^{2} \omega_{n_{j}}\left(k^{(j)}\right)$, the Hessian of $\omega_{n_{j}}$ at $k=k^{(j)}$, is (positive or negative) definite for each $j \in\{1, \ldots, N\}$. The smoothness assumption (A3) is needed to justify our Taylor expansions of $\omega_{n_{j}}$ near $k^{(j)}$. Bands $\omega_{n}$ are generally only Lipschitz continuous due to possible transversal intersections of bands and their numbering according to size [20]. Away from points of intersection or tangency bands can be shown to be actually analytic in $k$ by standard perturbation theory [18. The simplest situation when (A3) is satisfied is thus when each band $\omega_{n_{j}}$ is isolated near $k^{(j)}$, which is equivalent to $n_{1}=\ldots=n_{N}$ due to our ordering of bands according to size of $\omega_{n}(k)$ at each $k$.

Note that since each band $\omega_{n_{j}}$ has an extremum at $k=k^{(j)}$, we have $\partial_{k_{1}} \omega_{n_{j}}\left(k^{(j)}\right)=\partial_{k_{2}} \omega_{n_{j}}\left(k^{(j)}\right)=0$ for $j \in\{1, \ldots, N\}$. Assumption (A4) then guarantees that the leading order terms in the Taylor expansion of the band $\omega_{n_{j}}$ around $k=k^{(j)}$ are in fact quadratic.

The asymptotic expansion for the electrical field $E$ of gap solitons with $\omega$ in the gap and in the vicinity 
of the edge $\omega_{*}$ is expected [12, 13, to be of the following slowly varying envelope form

$$
\begin{aligned}
& \varepsilon \sum_{j=1}^{N} A_{j}(y) u_{n_{j}}\left(k^{(j)} ; x\right)+\varepsilon^{2} \psi^{(1)}(x)+\varepsilon^{3} \psi^{(2)}(x)+\mathcal{O}\left(\varepsilon^{4}\right), \\
& \omega=\omega_{*}+\Omega \varepsilon^{2}, \quad y=\varepsilon x, \quad 0<\varepsilon \ll 1,
\end{aligned}
$$

where $A_{j}: \mathbb{R}^{2} \rightarrow \mathbb{C}$ is a fast decaying smooth function and where $\Omega= \pm 1$. The sign of $\Omega$ is determined by the condition that $\omega_{*}+\varepsilon^{2} \Omega$ lies in the gap.

Performing a multiple scales analysis in the physical variables $(x, y)$ is impossible. The reason is that in order to solve the resulting equations at each order of the expansion, one has to ensure that inhomogeneous terms are orthogonal to the kernel of $L-\omega_{*}^{2} \eta$, i.e., to $u_{n_{j}}\left(k^{(j)} ;\right.$.) for all $j \in\{1, \ldots, N\}$. This orthogonality needs to be checked on the common period of those $u_{n_{j}}$. If, however, one of the components of $k^{(j)}$ is irrational, the corresponding $u_{n_{j}}$ is not even periodic and this approach fails similarly to [13. We therefore perform the asymptotic analysis in Bloch variables where all functions are $U$-periodic in $x$ and orthogonality conditions are always posed over $U$.

Let us define the Bloch transform $\mathcal{T}: E \mapsto \widetilde{E}$ and its inverse, cf. [8, Ch. 7], by

$$
\widetilde{E}(k ; x)=(\mathcal{T} E)(k ; x)=\sum_{K \in \Lambda^{*}} e^{\mathrm{i} K \cdot x} \widehat{E}(k+K), \quad E(x)=\left(\mathcal{T}^{-1} \widetilde{E}\right)(x)=\int_{\mathbb{B}} e^{\mathrm{i} k \cdot x} \widetilde{E}(k ; x) \mathrm{d} k
$$

for all $x, k \in \mathbb{R}^{2}$, where $\widehat{E}$ denotes the Fourier transform of $E$

$$
\widehat{E}(k):=(\mathcal{F} E)(k):=\frac{1}{(2 \pi)^{2}} \int_{\mathbb{R}^{2}} E(x) e^{-\mathrm{i} k \cdot x} \mathrm{~d} x, \quad E(x)=\left(\mathcal{F}^{-1} \widehat{E}\right)(k):=\int_{\mathbb{R}^{2}} \widehat{E}(k) e^{\mathrm{i} k \cdot x} \mathrm{~d} k .
$$

By definition we have the following properties of the Bloch transform

$$
\begin{array}{llrl}
\widetilde{E}(k ; x+R) & =\widetilde{E}(k ; x) & & \text { for all } R \in \Lambda, \\
\widetilde{E}(k+K ; x) & =e^{-\mathrm{i} K \cdot x} \widetilde{E}(k ; x) & & \text { for all } K \in \Lambda^{*} .
\end{array}
$$

Multiplication of two functions $f, g$ in physical space corresponds to convolution in Bloch space, i.e.,

$$
(\mathcal{T}(f g))(k ; x)=\int_{\mathbb{B}} \widetilde{f}(k-l ; x) \widetilde{g}(l ; x) \mathrm{d} l=:\left(\widetilde{f} *_{\mathbb{B}} \widetilde{g}\right)(k ; x),
$$

where (3.2) is used if $k-l \notin \mathbb{B}$. Especially, if $x \mapsto f(x)$ is $U$-periodic, then

$$
(\mathcal{T}(f g))(k ; x)=f(x)(\mathcal{T} g)(k ; x) .
$$

This can be easily checked by writing $f$ in the form of a Fourier series, i.e. $f(x)=\sum_{K \in \Lambda^{*}} c_{K} e^{\mathrm{i} K \cdot x}$, cf. [8, Ch. 7]. Exploiting this observation and applying the Bloch transform to (1.9) leads to

$$
\left(\widetilde{L}(k)-\omega^{2} \eta(x)\right) \widetilde{E}(k ; x)=\omega^{2} \widetilde{P}_{\mathrm{NL}}(k ; x) \text { for all } x, k \in \mathbb{R}^{2},
$$

where

$$
\widetilde{P}_{\mathrm{NL}}(k ; .)=\chi_{\mathrm{ci}}^{(3)} \mathcal{T}\left(2|E|^{2} E+E \cdot E \bar{E}\right)=\chi_{\mathrm{ci}}^{(3)}\left(2\left(E . *_{\mathbb{B}} \bar{E}\right) *_{\mathbb{B}} E+\left(E . *_{\mathbb{B}} E\right) *_{\mathbb{B}} \bar{E}\right),
$$

with $f . *_{\mathbb{B}} g:=\sum_{j} f_{j} *_{\mathbb{B}} g_{j}$ for vector valued $f, g$, while $f *_{\mathbb{B}} g$ is understood componentwise for scalar $f$ and vector valued $g$. By definition of the Bloch- and Fourier transformation one immediately finds

$$
\mathcal{T}\left(A_{j}(\varepsilon \cdot) e^{\mathrm{i} k^{(j)} \cdot(\cdot)}\right)(k ; x)=\varepsilon^{-2} \sum_{K \in \Lambda^{*}} \widehat{A}_{j}\left(\frac{1}{\varepsilon}\left(k-k^{(j)}+K\right)\right) e^{\mathrm{i} K \cdot x},
$$


so that the asymptotic ansatz (3.1) is transformed to

$$
\varepsilon \widetilde{\psi}^{(0)}(k ; x)+\varepsilon^{2} \widetilde{\psi}^{(1)}(k ; x)+\varepsilon^{3} \widetilde{\psi}^{(2)}(k ; x)+O\left(\varepsilon^{4}\right),
$$

where

$$
\widetilde{\psi}^{(0)}(k ; x)=\varepsilon^{-2} \sum_{j=1}^{N} \sum_{K \in \Lambda^{*}} \widehat{A}_{j}\left(\frac{1}{\varepsilon}\left(k-k^{(j)}+K\right)\right) e^{\mathrm{i} K \cdot x} p_{n_{j}}\left(k^{(j)} ; x\right) .
$$

Similarly to [13] and 14, due to the fast decay of the Bloch transform of $A_{j}$ in $k$, we approximate $\widehat{A}_{j}\left(\frac{1}{\varepsilon}\left(k-k^{(j)}+K\right)\right)$ by $\chi_{D_{\varepsilon^{r}}}\left(k-k^{(j)}+K\right) \widehat{A}_{j}\left(\frac{1}{\varepsilon}\left(k-k^{(j)}+K\right)\right)$ for some $r \in(0,1)$, where $\chi_{S}$ is the indicator function of a set $S$, $D_{\delta}:=B_{\delta}(0)$ with $B_{\delta}(z):=\left\{k \in \mathbb{R}^{2}:|k-z|<\delta\right\}$ for $\delta>0, z \in \mathbb{R}^{2}$.

We will therefore introduce the approximation

$$
\widetilde{E}(k ; x)=\varepsilon^{-1} \widetilde{E}^{(0)}(k ; x)+\widetilde{E}^{(1)}(k ; x)+\varepsilon \widetilde{E}^{(2)}(k ; x)+O\left(\varepsilon^{2}\right)
$$

with

$$
\widetilde{E}^{(0)}(k ; x)=\sum_{j=1}^{N} \sum_{K \in \Lambda^{*}} \chi_{D_{\varepsilon^{r}}}\left(k-k^{(j)}+K\right) \widehat{A}_{j}\left(\frac{1}{\varepsilon}\left(k-k^{(j)}+K\right)\right) e^{\mathrm{i} K \cdot x} p_{n_{j}}\left(k^{(j)} ; x\right)
$$

for all $k \in \mathbb{B}$ and $x \in \mathbb{R}^{2}$. In the following we will use the notation $K^{m}=m_{1} b^{(1)}+m_{2} b^{(2)} \in \Lambda^{*}$ for $m \in \mathbb{Z}^{2}$ for convenience. As an abbreviation we let $\ell^{(j, m)}(k):=\frac{1}{\varepsilon}\left(k-k^{(j)}+K^{m}\right)$ for $k \in \mathbb{R}^{2}$ and $m \in \mathbb{Z}^{2}$, so that $\widetilde{E}^{(0)}$ is given as

$$
\widetilde{E}^{(0)}(k ; x)=\sum_{j=1}^{N} \sum_{m \in \mathbb{Z}^{2}} \chi_{D_{\varepsilon} r}\left(\ell^{(j, m)}(k)\right) \widehat{A}_{j}\left(\ell^{(j, m)}(k)\right) e^{\mathrm{i} K^{m} \cdot x} p_{n_{j}}\left(k^{(j)} ; x\right) .
$$

Note that $\widetilde{E}^{(0)}(. ; x)$ is supported on a set of (for sufficiently small $\varepsilon$ ) disjoint balls $B_{\varepsilon^{r}}\left(k^{(j)}-K^{m}\right), j \in$ $\{1, \ldots, N\}, m \in \mathbb{Z}^{2}$.

3.2. Formal asymptotic analysis. Let us proceed with a formal asymptotic analysis of (1.9). First, we consider $k$ close to $k^{(j)}-K^{m}$, i.e., $k \in B_{\varepsilon^{r}}\left(k^{(j)}-K^{m}\right)$ for some $j \in\{1, \ldots, N\}, m \in \mathbb{Z}^{2}$. Then

$$
\begin{aligned}
\widetilde{L}(k) & =\widetilde{L}\left(k^{(j)}-K^{m}+\varepsilon \ell^{(j, m)}(k)\right) \\
& =\widetilde{L}\left(k^{(j)}-K^{m}\right)+\varepsilon \ell^{(j, m)}(k) \cdot \partial_{k} \widetilde{L}\left(k^{(j)}-K^{m}\right)+\frac{1}{2} \varepsilon^{2} Q\left(\ell^{(j, m)}(k)\right),
\end{aligned}
$$

where we have used the fact that the second derivatives of $\widetilde{L}$ are constant in $k$, see (2.8), and where

$$
\begin{aligned}
\ell^{(j, m)}(k) \cdot \partial_{k} \widetilde{L}\left(k^{(j)}-K^{m}\right) & =\sum_{i=1}^{2} \ell_{i}^{(j, m)}(k) \partial_{k_{i}} \widetilde{L}\left(k^{(j)}-K^{m}\right), \text { and } \\
Q\left(\ell^{(j, m)}(k)\right) & =\sum_{a, b=1}^{2} \ell_{a}^{(j, m)}(k) \ell_{b}^{(j, m)}(k) \partial_{k_{a}, k_{b}}^{2} \tilde{L} .
\end{aligned}
$$

Using (3.3), (3.4), (3.5) and $\omega=\omega_{*}+\Omega \varepsilon^{2}$, we get a hierarchy of equations at each power of $\varepsilon$ for $x \in U$ and $k \in B_{\varepsilon^{r}}\left(k^{(j)}-K^{m}\right)$. We now study the equations related to $\varepsilon^{-1}, \varepsilon^{0}, \varepsilon^{1}$ under the condition that the nonlinear term contributes to $\varepsilon^{1}$, which is confirmed later in (3.14).

$\mathbf{O}\left(\varepsilon^{-1}\right)$ : The resulting equation is

$$
\begin{aligned}
& \widehat{A}_{j}\left(\ell^{(j, m)}(k)\right)\left(\widetilde{L}\left(k^{(j)}-K^{m}\right)-\omega_{*}^{2} \eta(x)\right)\left(p_{n_{j}}\left(k^{(j)} ; x\right) e^{\mathrm{i} K^{m} \cdot x}\right) \\
& \quad=\widehat{A}_{j}\left(\ell^{(j, m)}(k)\right) e^{\mathrm{i} K^{m} \cdot x}\left(\widetilde{L}\left(k^{(j)}\right)-\omega_{*}^{2} \eta(x)\right) p_{n_{j}}\left(k^{(j)} ; x\right) \stackrel{!}{=} 0 .
\end{aligned}
$$


This holds by the definitions of $\omega_{*}=\omega_{n_{j}}\left(k^{(j)}\right)$ and $p_{n_{j}}\left(k^{(j)} ;.\right)$.

$\mathbf{O}(\mathbf{1})$ : The resulting equation is

$$
\begin{aligned}
& \left(\widetilde{L}\left(k^{(j)}-K^{m}\right)-\omega_{*}^{2} \eta(x)\right) \widetilde{E}^{(1)}(k ; x) \\
& \quad=-\widehat{A}_{j}\left(\ell^{(j, m)}(k)\right)\left(\ell^{(j, m)}(k) \cdot \partial_{k} \widetilde{L}\left(k^{(j)}-K^{m}\right)\right)\left(p_{n_{j}}\left(k^{(j)} ; x\right) e^{\mathrm{i} K^{m} \cdot x}\right) \\
& \quad=-\widehat{A}_{j}\left(\ell^{(j, m)}(k)\right) e^{\mathrm{i} K^{m} \cdot x}\left(\ell^{(j, m)}(k) \cdot \partial_{k} \widetilde{L}\left(k^{(j)}\right)\right) p_{n_{j}}\left(k^{(j)} ; x\right) \stackrel{!}{=} 0 .
\end{aligned}
$$

Using (2.6), the solution is found to be

$$
\widetilde{E}^{(1)}(k ; x)=\widehat{A}_{j}\left(\ell^{(j, m)}(k)\right) e^{\mathrm{i} K^{m} \cdot x}\left(\ell^{(j, m)}(k) \cdot \partial_{k} p_{n_{j}}\left(k^{(j)} ; x\right)\right),
$$

where $\ell^{(j, m)}(k) \cdot \partial_{k} p_{n_{j}}\left(k^{(j)} ; x\right)=\sum_{i=1}^{2} \ell_{i}^{(j, m)}(k) \partial_{k_{i}} p_{n_{j}}\left(k^{(j)} ; x\right)$.

$\mathbf{O}(\varepsilon)$ : The contribution of $\widetilde{L}(k) \widetilde{E}$ is

$$
\begin{aligned}
& \left(\widetilde{L}\left(k^{(j)}-K^{m}\right)-\omega_{*}^{2} \eta(x)\right) \widetilde{E}^{(2)}(k ; x) \\
& \quad+\frac{1}{2} Q\left(\ell^{(j, m)}\right) \widetilde{E}^{(0)}(k ; x)-2 \omega_{*} \Omega \eta(x) \widetilde{E}^{(0)}(k ; x) \\
& \quad+\left(\ell^{(j, m)}(k) \cdot \partial_{k} \widetilde{L}\left(k^{(j)}-K^{m}\right)\right) \widetilde{E}^{(1)}(k ; x) .
\end{aligned}
$$

By insertion of the previous results this gives (for $k \in B_{\varepsilon^{r}}\left(k^{(j)}-K^{m}\right)$ )

$$
\begin{aligned}
& \left(\widetilde{L}\left(k^{(j)}-K^{m}\right)-\omega_{*}^{2} \eta(x)\right) \widetilde{E}^{(2)}(k ; x) \\
& +\left[\frac{1}{2} Q\left(\ell^{(j, m)}(k)\right) p_{n_{j}}\left(k^{(j)} ; x\right)-2 \omega_{*} \Omega \eta(x) p_{n_{j}}\left(k^{(j)} ; x\right)\right. \\
& \left.+\left(\ell^{(j, m)}(k) \cdot \partial_{k} \widetilde{L}\left(k^{(j)}-K^{m}\right)\right)\left(\ell^{(j, m)}(k) \cdot \partial_{k} p_{n_{j}}\left(k^{(j)} ; x\right)\right)\right] \widehat{A}_{j}\left(\ell^{(j, m)}(k)\right) e^{\mathrm{i} K^{m} \cdot x} \\
& \stackrel{!}{=} \omega_{*}^{2} \chi_{\mathrm{ci}}^{(3)}(x) \frac{1}{\varepsilon^{4}}\left(2\left(\widetilde{E}^{(0)} \cdot *_{\mathbb{B}} \widetilde{\widetilde{E^{(0)}}}\right) *_{\mathbb{B}} \widetilde{E}^{(0)}+\left(\widetilde{E}^{(0)} \cdot *_{\mathbb{B}} \widetilde{E}^{(0)}\right) *_{\mathbb{B}} \widetilde{\widetilde{E^{(0)}}}\right)(k ; x) \\
& =: \omega_{*}^{2} \chi_{\mathrm{ci}}^{(3)}(x) \widetilde{G}_{j}(k, x) \text {. }
\end{aligned}
$$

The remainder of the section is devoted to the analysis of the structure of $\widetilde{G}_{j}$ in (3.7) and to the derivation of a solvability condition for (3.7).

Let us first analyze the nonlinearity. The convolutions in (3.7) can be expanded into the form

$$
\widetilde{E}_{a}^{(0)} *_{\mathbb{B}} \widetilde{E}_{b}^{(0)} *_{\mathbb{B}} \widetilde{\widetilde{E_{c}^{(0)}}}=\sum_{\alpha, \beta, \gamma=1}^{N} \xi_{\alpha, a} *_{\mathbb{B}} \xi_{\beta, b} *_{\mathbb{B}} \xi_{\gamma, c}^{\times},
$$

where $a, b, c \in\{1,2,3\}$, and functions $\xi_{\alpha, a}$ and $\xi_{\alpha, a}^{\times}$are given by

$$
\begin{aligned}
& \xi_{\alpha, a}(k ; x):=p_{n_{\alpha}, a}\left(k^{(\alpha)} ; x\right) \sum_{z \in \mathbb{Z}^{2}} \chi_{D_{\varepsilon^{r}}}\left(k-k^{(\alpha)}+K^{z}\right) \widehat{A}_{\alpha}\left(\frac{1}{\varepsilon}\left(k-k^{(\alpha)}+K^{z}\right)\right) e^{\mathrm{i} K^{z} \cdot x}, \\
& \xi_{\alpha, a}^{\times}(k ; x):=\overline{p_{n_{\alpha}, a}}\left(k^{(\alpha)} ; x\right) \sum_{z \in \mathbb{Z}^{2}} \chi_{D_{\varepsilon^{r}}}\left(k+k^{(\alpha)}-K^{z}\right) \widehat{A}_{\alpha}\left(\frac{1}{\varepsilon}\left(k+k^{(\alpha)}-K^{z}\right)\right) e^{-\mathrm{i} K^{z} \cdot x} .
\end{aligned}
$$

Note that (3.8) represents all the nonlinear terms in (3.7) due to commutativity of $*_{\mathbb{B}}$. The summands in (3.8) have the form

$$
\left(\xi_{\alpha, a} *_{\mathbb{B}} \xi_{\beta, b} *_{\mathbb{B}} \xi_{\gamma, c}^{\times}\right)(k ; x)=\sum_{n, o, q \in \mathbb{Z}^{2}} g_{n o q}(k ; x)=\sum_{n \in M_{\alpha}^{(2)}, o \in M_{\beta}^{(2)}, q \in M_{\gamma}} g_{n o q}(k ; x),
$$


where (with indices $\alpha, \beta, \gamma, a, b, c$ suppressed)

$$
\begin{array}{r}
g_{n o q}(k ; x)=e^{\mathrm{i}\left(K^{n}+K^{o}-K^{q}\right) \cdot x} p_{n_{\alpha}, a}\left(k^{(\alpha)} ; x\right) p_{n_{\beta}, b}\left(k^{(\beta)} ; x\right) \overline{p_{n_{\gamma}, c}}\left(k^{(\gamma)} ; x\right) \\
\int_{\mathbb{B}} \int_{\mathbb{B}} \chi_{D_{\varepsilon^{r}}}\left(k-t-k^{(\alpha)}+K^{n}\right) \widehat{A}_{\alpha}\left(\frac{1}{\varepsilon}\left(k-t-k^{(\alpha)}+K^{n}\right)\right) \\
\quad \times \chi_{D_{\varepsilon^{r}}}\left(t-s-k^{(\beta)}+K^{o}\right) \widehat{A}_{\beta}\left(\frac{1}{\varepsilon}\left(t-s-k^{(\beta)}+K^{o}\right)\right) \\
\quad \times \chi_{D_{\varepsilon^{r}}}\left(s+k^{(\gamma)}-K^{q}\right) \widehat{\bar{A}}_{\gamma}\left(\frac{1}{\varepsilon}\left(s+k^{(\gamma)}-K^{q}\right)\right) \mathrm{d} s \mathrm{~d} t
\end{array}
$$

and with

$$
\begin{aligned}
M_{\gamma} & =\left\{z \in \mathbb{Z}^{2}: k-k^{(\gamma)}+K^{z} \in B_{\varepsilon^{r}}(0) \text { for some } k \in \mathbb{B} \text { and all } \varepsilon>0\right\}, \\
M_{b}^{(2)} & =\left\{z \in \mathbb{Z}^{2}: k-k^{(b)}+K^{z} \in B_{\varepsilon^{r}}(0) \text { for some } k \in \mathbb{B}+\mathbb{B} \text { and all } \varepsilon>0\right\}
\end{aligned}
$$

for $b \in\{\alpha, \beta\}$. The truncation of the series in (3.9) comes from the fact that for $s, t, k \in \mathbb{B}$ we have $t-s \in \mathbb{B}+\mathbb{B}$ and $k-t \in \mathbb{B}+\mathbb{B}$ so that the three characteristic functions in (3.10) can be nonzero only for $n \in M_{\alpha}^{(2)}, o \in M_{\beta}^{(2)}$, and $q \in M_{\gamma}$. More precisely, this is seen as follows.

Only those combinations of $n, o, q$ which produce nonzero values of all the three characteristic functions in (3.10) and of the function $\chi_{D_{\varepsilon} r}\left(.-k^{(j)}+K^{m}\right)$ in (3.7) for given $j$ and some $k, t, s \in \mathbb{B}$ are of relevance. Firstly, $\chi_{D_{\varepsilon} r}\left(s+k^{(\gamma)}-K^{q}\right)$ is nonzero for some $s \in \mathbb{B}$ and for arbitrary $\varepsilon>0$ if and only if $s_{0}:=-k^{(\gamma)}+K^{q} \in \overline{\mathbb{B}}$ (the closure of $\mathbb{B}$ ) for some $q \in \mathbb{Z}^{2}$, which is equivalent to

$$
q \in M_{\gamma}
$$

Secondly, for a fixed $q$ the factor $\chi_{D_{\varepsilon^{r}}}\left(t-s-k^{(\beta)}+K^{o}\right)$ is nonzero for all $\varepsilon>0$ and some $t \in \mathbb{B}$ and $s$ obtained in the first step if and only if $t_{0}:=s_{0}+k^{(\beta)}-K^{o} \in \overline{\mathbb{B}}$, i.e.,

$$
k^{(\beta)}-k^{(\gamma)}+K^{q}-K^{o} \in \overline{\mathbb{B}} .
$$

This can always be satisfied by a choice of $o \in M_{\beta}^{(2)}$. Finally, for fixed $q$ and $o$ we need that $\chi_{D_{\varepsilon} r}\left(k-t-k^{(\alpha)}+\right.$ $\left.K^{n}\right)$ does not vanish for some $k \in \mathbb{B}$ with $k-k^{(j)}+K^{m} \in D_{\varepsilon^{r}}$ and all $\varepsilon>0$, where this latter restriction is due to the restriction $k \in B_{\varepsilon^{r}}\left(k^{(j)}-K^{m}\right)$ in (3.7). In other words, we need that $k_{0}:=k^{(j)}-K^{m} \in \overline{\mathbb{B}}$ and $0=k_{0}-t_{0}-k^{(\alpha)}+K^{n}$, i.e.

$$
k^{(\alpha)}+k^{(\beta)}-k^{(\gamma)}+K^{q}-K^{o}-K^{n}=k^{(j)}-K^{m} \in \overline{\mathbb{B}}
$$

for some $n \in \mathbb{Z}^{2}$. In fact, all solutions for $n$ of (3.13) lie in $M_{\alpha}^{(2)}$.

In summary, for $\alpha, \beta, \gamma \in\{1, \ldots, N\}$ the term $g_{n o q}$ is nonzero in (3.10) if $n, o, q$ satisfy (3.11), (3.12) and (3.13). So the term $\xi_{\alpha, a} *_{\mathbb{B}} \xi_{\beta, b} *_{\mathbb{B}} \xi_{\gamma, c}^{\times}$enters $\widetilde{G}_{j}$ provided

$$
\mathcal{A}_{\alpha, \beta, \gamma, j}:=\left\{(n, o, q) \in\left(\mathbb{Z}^{2}\right)^{3}: n \in M_{\alpha}^{(2)}, o \in M_{\beta}^{(2)}, q \in M_{\gamma} \text { and (3.12), (3.13) hold }\right\}
$$

is nonempty for some $m \in M_{j}$. Note that we omitted an index $m$ in this definition, because the set is either nonempty or empty for all $m \in M_{j}$. Indeed, if $m$ is one index that meets the requirements with $(n, o, q)$ and $z$ is any other index in $M_{j}$, then $z$ meets the requirements for $(n+m-z, o, q) . \mathcal{A}_{\alpha, \beta, \gamma, j}$ can be constructed by a computer code that scans all possible combinations of $n, o, q$. This will be discussed in Section 3.4 ,

Due to the characteristic function, the integration domains in (3.10) can be reduced to $s \in B_{\varepsilon^{r}}\left(-k^{(\gamma)}+\right.$ $\left.K^{q}\right) \cap \mathbb{B}$ and $t \in B_{2 \varepsilon^{r}}\left(k^{(\beta)}-k^{(\gamma)}-K^{o}+K^{q}\right) \cap \mathbb{B}$. Now we introduce the change of variables $\tilde{s}:=\left(s+k^{(\gamma)}-K^{q}\right) / \varepsilon$ 
and $\tilde{t}:=\left(t-k^{(\beta)}+k^{(\gamma)}+K^{o}-K^{q}\right) / \varepsilon$ to get

$$
\begin{aligned}
g_{n o q}(k ; x) & =\varepsilon^{4} e^{\mathrm{i}\left(K^{n}+K^{o}-K^{q}\right) \cdot x} p_{n_{\alpha}, a}\left(k^{(\alpha)} ; x\right) p_{n_{\beta}, b}\left(k^{(\beta)} ; x\right) \overline{p_{n_{\gamma}, c}}\left(k^{(\gamma)} ; x\right) \\
\times & \int_{D_{2 \varepsilon^{r-1}} \cap \frac{\mathbb{B}-k^{(\beta)}+k^{(\gamma)}+K^{o}-K^{q}}{\varepsilon}{ }_{D_{\varepsilon^{r-1}} \cap \frac{\mathbb{B}+k^{(\gamma)}-K^{q}}{\varepsilon}} \chi_{D_{\varepsilon^{r-1}}}\left(\frac{k-\left(k^{(\alpha)}+k^{(\beta)}-k^{(\gamma)}\right)+K^{n}+K^{o}-K^{q}}{\varepsilon}-\tilde{t}\right)} \\
& \times \widehat{A}_{\alpha}\left(\frac{k-\left(k^{(\alpha)}+k^{(\beta)}-k^{(\gamma)}\right)+K^{n}+K^{o}-K^{q}}{\varepsilon}-\tilde{t}\right) \chi_{D_{\varepsilon^{r-1}}}(\tilde{t}-\tilde{s}) \widehat{A}_{\beta}(\tilde{t}-\tilde{s}) \chi_{D_{\varepsilon^{r-1}}}(\tilde{s}) \widehat{\bar{A}}_{\gamma}(\tilde{s}) \mathrm{d} \tilde{s} \mathrm{~d} \tilde{t} .
\end{aligned}
$$

The factor $\varepsilon^{4}$ in this formula shows that $\widetilde{G}_{j}=O(1)$ as required for the consistent asymptotic expansion. If (3.13) is satisfied, (3.14) becomes

$$
\begin{aligned}
g_{n o q}(k ; x) & =\varepsilon^{4} e^{\mathrm{i}\left(k^{(\alpha)}+k^{(\beta)}-k^{(\gamma)}-k^{(j)}+K^{m}\right) \cdot x} p_{n_{\alpha}, a}\left(k^{(\alpha)} ; x\right) p_{n_{\beta}, b}\left(k^{(\beta)} ; x\right) \overline{p_{n_{\gamma}, c}}\left(k^{(\gamma)} ; x\right) \\
\times & \int_{D_{2 \varepsilon^{r-1}} \cap \frac{\mathbb{B}-k(\beta)+k^{(\gamma)}+K^{o}-K^{q}}{\varepsilon}} \int_{D_{\varepsilon^{r-1}} \cap \frac{\mathbb{B}+k(\gamma)-K^{q}}{\varepsilon}} \chi_{D_{\varepsilon^{r-1}}}\left(\frac{k-k^{(j)}+K^{m}}{\varepsilon}-\tilde{t}\right) \\
& \times \widehat{A}_{\alpha}\left(\frac{k-k^{(j)}+K^{m}}{\varepsilon}-\tilde{t}\right) \chi_{D_{\varepsilon^{r-1}}}(\tilde{t}-\tilde{s}) \widehat{A}_{\beta}(\tilde{t}-\tilde{s}) \chi_{D_{\varepsilon^{r-1}}}(\tilde{s}) \widehat{\bar{A}}_{\gamma}(\tilde{s}) \mathrm{d} \tilde{s} \mathrm{~d} \tilde{t}
\end{aligned}
$$

for $k \in B_{\varepsilon^{r}}\left(k^{(j)}-K^{m}\right)$. As we show in Remark 3.1 summing, for fixed $k, j, m$, the terms (3.15) over $(n, o, q) \in \mathcal{A}_{\alpha, \beta, \gamma, j}$ yields a double convolution integral in $\tilde{s}, \tilde{t}$ over the full discs $D_{2 \varepsilon^{r-1}}$ and $D_{\varepsilon^{r-1}}$, i.e.,

$$
\begin{aligned}
& \left(\xi_{\alpha, a} *_{\mathbb{B}} \xi_{\beta, b} *_{\mathbb{B}} \xi_{\gamma, c}^{\times}\right)(k ; x)=\varepsilon^{4} e^{\mathrm{i}\left(k^{(\alpha)}+k^{(\beta)}-k^{(\gamma)}-k^{(j)}+K^{m}\right) \cdot x} p_{n_{\alpha}, a}\left(k^{(\alpha)} ; x\right) p_{n_{\beta}, b}\left(k^{(\beta)} ; x\right) \overline{p_{n_{\gamma}, c}}\left(k^{(\gamma)} ; x\right) \\
& \quad \times \quad \iint_{D_{2^{r-1}}} \chi_{D_{\varepsilon^{r-1}}}\left(\ell^{(j, m)}(k)-\tilde{t}\right) \widehat{A}_{\alpha}\left(\ell^{(j, m)}(k)-\tilde{t}\right) \chi_{D_{\varepsilon^{r-1}}}(\tilde{t}-\tilde{s}) \widehat{A}_{\beta}(\tilde{t}-\tilde{s}) \chi_{D_{\varepsilon^{r-1}}}(\tilde{s}) \widehat{\bar{A}}_{\gamma}(\tilde{s}) \mathrm{d} \tilde{s} \mathrm{~d} \tilde{t} \\
& \quad=: \varepsilon^{4} e^{\mathrm{i}\left(-k^{(j)}+K^{m}\right) \cdot x} u_{n_{\alpha}, a}\left(k^{(\alpha)} ; x\right) u_{n_{\beta}, b}\left(k^{(\beta)} ; x\right) \overline{u_{n_{\gamma}, c}}\left(k^{(\gamma)} ; x\right) \tilde{h}_{\alpha, \beta, \gamma}^{(\varepsilon)}\left(\ell^{(j, m)}(k)\right)
\end{aligned}
$$

for $k \in B_{\varepsilon^{r}}\left(k^{(j)}-K^{m}\right)$. Here we have used $u_{n_{\alpha}, a}\left(k^{(\alpha)} ; x\right)=p_{n_{\alpha}, a}\left(k^{(\alpha)} ; x\right) e^{\mathrm{i} k^{(\alpha)} \cdot x}$, etc., and we defined $\tilde{h}_{\alpha, \beta, \gamma}^{(\varepsilon)}\left(\ell^{(j, m)}(k)\right)$ as an abbreviation for the integral on the right hand side.

REMARK 3.1. To show that the sum of $g_{\text {noq }}$ over $(n, o, q) \in \mathcal{A}_{\alpha, \beta, \gamma, j}$ yields a double convolution integral over full discs, let us first note that the definitions of $M_{\gamma}$ and $M_{\beta}^{(2)}$ ensure

$$
\bigcup_{q \in M_{\gamma}}\left(\left(\mathbb{B}+k^{(\gamma)}-K^{q}\right) \cap D_{\varepsilon^{r}}\right)=D_{\varepsilon^{r}}
$$

and

$$
\bigcup_{o \in M_{\beta}^{(2)}}\left(\left(\mathbb{B}-k^{(\beta)}+k^{(\gamma)}+K^{o}-K^{q}\right) \cap D_{2 \varepsilon^{r}}\right)=D_{2 \varepsilon^{r}}
$$

These are obvious when $k^{(\gamma)} \in \operatorname{int}(\mathbb{B})$ and $k^{(\beta)}, k^{(\gamma)} \in \operatorname{int}(\mathbb{B})$, respectively, because then $M_{\gamma}=M_{\beta}^{(2)}=\left\{(0,0)^{T}\right\}$. But when $k^{(\gamma)} \in \partial \mathbb{B}$, then only a fraction of $-k^{(\gamma)}+D_{\varepsilon^{r}}$ lies in $\mathbb{B}$ (in our example with a hexagonal $\mathbb{B}$ the fraction is a half unless $k^{(\gamma)}$ is a vertex of $\mathbb{B}$, in which case it is a third) and the rest lies in periodicity cells centered at neighboring reciprocal lattice points. Each point $\ell$ in this rest is therefore mapped to $\mathbb{B}$ via $\ell+K^{q}$ with some $q \in M_{\gamma}$, and we thus have (3.17). By an analogous argument, observing that $k^{(\beta)}-\left(k^{(\gamma)}-K^{q}\right) \in \mathbb{B}+D_{\varepsilon^{r}}$ for all $q \in M_{\gamma}$, we get (3.18) from the definition of $M_{\beta}^{(2)}$.

Let us now assume (3.13) and show that for each $K^{q}$ fixed, i.e. for each fixed integration domain in the inner integral in (3.14), the sum of $g_{n o q}$ over $(n, o, q) \in \mathcal{A}_{\alpha, \beta, \gamma, j}$ yields an integration over the full disc $D_{2 \varepsilon^{r}}$ in the outer integral. If this were not the case, i.e. if $\exists \ell \in D_{2 \varepsilon^{r}}$ such that $\ell \notin \mathbb{B}-k^{(\beta)}+k^{(\gamma)}+K^{o}-K^{q}$ 
for any such $(n, o, q) \in \mathcal{A}_{\alpha, \beta, \gamma, j}$, then by (3.18) there would be $o \in M_{\beta}^{(2)}$ such that $(n, o, q) \notin \mathcal{A}_{\alpha, \beta, \gamma, j}$ while (3.12) and (3.13) are satisfied. This is a contradiction to the definition of $\mathcal{A}_{\alpha, \beta, \gamma, j}$. After that we sum over all $q \in M_{\gamma}$ and the result follows from (3.14).

We now write the $d$-th component $(d \in\{1,2,3\})$ of $\widetilde{G}_{j}$ as

$$
\widetilde{G}_{j, d}(k ; x)=\varepsilon^{-4} \chi_{D_{\varepsilon} r}\left(k-k^{(j)}+K^{m}\right) \sum_{a, b, c=1}^{3} \Gamma_{a, b, c}^{(d)}\left(\widetilde{E}_{a}^{(0)} *_{\mathbb{B}} \widetilde{E}_{b}^{(0)} *_{\mathbb{B}} \widetilde{\overline{E_{c}^{(0)}}}\right)(k ; x),
$$

where the integer coefficients $\Gamma_{a, b, c}^{(d)}$ can be easily derived from (1.7). In detail we have $\Gamma_{1,1,1}^{(1)}=\Gamma_{2,2,2}^{(2)}=\Gamma_{3,3,3}^{(3)}=$ $3, \Gamma_{1,2,2}^{(1)}=\Gamma_{2,1,2}^{(1)}=\Gamma_{1,3,3}^{(1)}=\Gamma_{3,1,3}^{(1)}=\Gamma_{2,2,1}^{(1)}=\Gamma_{3,3,1}^{(1)}=1, \Gamma_{1,2,1}^{(2)}=\Gamma_{2,1,1}^{(2)}=\Gamma_{3,2,3}^{(2)}=\Gamma_{2,3,3}^{(2)}=\Gamma_{1,1,2}^{(2)}=\Gamma_{3,3,2}^{(2)}=1$, $\Gamma_{1,3,1}^{(3)}=\Gamma_{3,1,1}^{(3)}=\Gamma_{2,3,2}^{(3)}=\Gamma_{3,2,2}^{(3)}=\Gamma_{1,1,3}^{(3)}=\Gamma_{2,2,3}^{(3)}=1$, and the remaining $\Gamma_{a, b, c}^{(d)}$ are zero. Finally, using (3.16), we get for $k \in B_{\varepsilon^{r}}\left(k^{(j)}-K^{m}\right)$

$$
\begin{array}{r}
\widetilde{G}_{j, d}(k ; x)=e^{\mathrm{i}\left(-k^{(j)}+K^{m}\right) \cdot x} \sum_{a, b, c=1}^{3} \Gamma_{a, b, c}^{(d)} \sum_{\substack{\alpha, \beta, \gamma \in\{1, \ldots, N\} \text { s.t. } \\
\mathcal{A}_{\alpha, \beta, \gamma, j \neq \emptyset}}} u_{n_{\alpha}, a}\left(k^{(\alpha)} ; x\right) u_{n_{\beta}, b}\left(k^{(\beta)} ; x\right) \\
\times \overline{u_{n_{\gamma}, c}}\left(k^{(\gamma)} ; x\right) \tilde{h}_{\alpha, \beta, \gamma}^{(\varepsilon)}\left(\ell^{(j, m)}(k)\right) .
\end{array}
$$

In order to make the discussion of the asymptotic hierarchy complete, we also have to consider the part of the $k$-domain outside the neighborhoods of $k^{(j)}$. For $k \in \mathbb{B}$ such that $k-k^{(j)}+K^{m} \in \mathbb{B} \backslash D_{\varepsilon^{r}}$ for all $m \in M_{j}$ we have $\left(\widetilde{L}\left(k^{(j)}-K^{m} ; x\right)-\omega_{*}^{2} \eta(x)\right) \widetilde{E}^{(l)}(k ; x)=0$ for $l \in\{0,1\}$ so that $\widetilde{E}^{(0)}(k ;.) \equiv \widetilde{E}^{(1)}(k ;.) \equiv 0$ for such $k$.

3.3. Coupled mode equations. We return now to equation (3.7). Due to the Fredholm alternative the existence of $\Lambda$-periodic solutions $\widetilde{E}^{(2)}$ of equation (3.7) is equivalent to $L^{2}$-orthogonality of (3.7) to $p_{n_{j}}\left(k^{(j)} ; x\right) e^{\mathrm{i} K^{m} \cdot x}$, which needs to be ensured for all $m \in M_{j}$ and $j \in\{1, \ldots, N\}$. The range of $\ell^{(j, m)}$ is a different section of the disc $D_{\varepsilon^{r-1}}$ for each $m \in M_{j}$. This section is a $\left(1 /\left|M_{j}\right|\right)$-th of the full disc so that these $\left|M_{j}\right|$ equations actually build one equation in $\ell \in D_{\varepsilon^{r-1}}$. Figure 3.1 shows these sections for two example points $k^{(j)}$. One example is for $\left|M_{j}\right|=2$ and the other one for $\left|M_{j}\right|=3$.

When imposing the orthogonality condition, the common factor $e^{\mathrm{i} K^{m} \cdot x}$ of the right hand side of (3.7) is canceled in the complex inner product with $p_{n_{j}}\left(k^{(j)} ; x\right) e^{\mathrm{i} K^{m} \cdot x}$, so that the same solvability condition holds for all $m \in M_{j}$. Using (3.7), (2.5), and (2.7) (with $n_{*}$ and $k_{*}$ replaced by $n_{j}$ and $k^{(j)}$ ), we obtain

$$
\Omega \widehat{A}_{j}(\ell)-\frac{1}{2}\left(\ell_{1}^{2} \partial_{k_{1}}^{2} \omega_{n_{j}}\left(k^{(j)}\right)+\ell_{2}^{2} \partial_{k_{2}}^{2} \omega_{n_{j}}\left(k^{(j)}\right)+2 \ell_{1} \ell_{2} \partial_{k_{1}, k_{2}}^{2} \omega_{n_{j}}\left(k^{(j)}\right)\right) \widehat{A}_{j}(\ell)+\widehat{\mathcal{N}}_{j}(\ell)=0
$$

for $\ell \in D_{\varepsilon^{r-1}}$, where

$$
\begin{aligned}
& \widehat{\mathcal{N}}_{j}(\ell)=\frac{\omega_{*}}{2}\left\langle\chi_{\mathrm{ci}}^{(3)}(.) \widetilde{G}_{j}(\ell ; .), p_{n_{j}}\left(k^{(j)} ; .\right) e^{-\mathrm{i} K^{m} \cdot(\cdot)}\right\rangle \\
& =\frac{\omega_{*}}{2} \sum_{a, b, c, d=1}^{3} \Gamma_{a, b, c}^{(d)} \sum_{\substack{\alpha, \beta, \gamma \in\{1, \ldots, N\} \text { s.t. } \\
\mathcal{A}_{\alpha, \beta, \gamma, j} \neq \emptyset}} \int_{U} \chi_{\mathrm{ci}}^{(3)}(x) u_{n_{\alpha}, a}\left(k^{(\alpha)} ; x\right) u_{n_{\beta}, b}\left(k^{(\beta)} ; x\right) \\
& =: \sum_{\substack{\alpha, \beta, \gamma \in\{1, \ldots, N\} \text { s.t. } \\
\mathcal{A}_{\alpha, \beta, \gamma, j \neq \emptyset}}} I_{\alpha, \beta, \gamma, j} \tilde{h}_{\alpha, \beta, \gamma}^{(\varepsilon)}(\ell), \\
& \times \overline{u_{n_{\gamma}, c}}\left(k^{(\gamma)} ; x\right) \overline{u_{n_{j}, d}}\left(k^{(j)} ; x\right) \mathrm{d} x \tilde{h}_{\alpha, \beta, \gamma}^{(\varepsilon)}(\ell)
\end{aligned}
$$


(a)

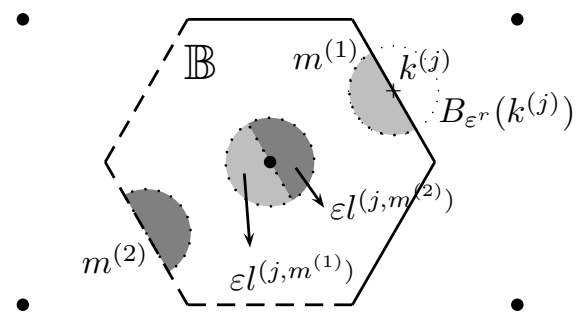

(b)

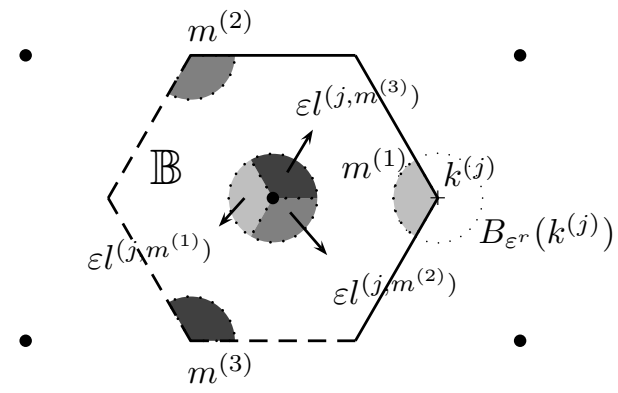

Fig. 3.1: Two example points $k^{(j)}$ in the case of the hexagonal lattice and the corresponding ranges of $\varepsilon \ell^{(j, m)}$ for all $m \in M_{j}$. In (a) we have $M_{j}=\left\{(0,0)^{T},(1,1)^{T}\right\}=:\left\{m^{(1)}, m^{(2)}\right\}$ and in (b) $M_{j}=\left\{(0,0)^{T},(0,1)^{T},(1,1)^{T}\right\}=:\left\{m^{(1)}, m^{(2)}, m^{(3)}\right\}$. The shaded sections along the boundary of $\mathbb{B}$ are those $k \in \mathbb{B}$ for which $\chi_{D_{\varepsilon} r}\left(k-k^{(j)}+K^{m}\right) \neq 0$ for the $m \in M_{j}$ written next to the respective section.

i.e. with (1.7) and the definition of $\Gamma$ in (3.19)

$$
\begin{aligned}
I_{\alpha, \beta, \gamma, j}:= & \frac{\omega_{*}}{2} \sum_{a, b, c, d=1}^{3} \Gamma_{a, b, c}^{(d)} \int_{U} \chi_{\mathrm{ci}}^{(3)} u_{n_{\alpha}, a}\left(k^{(\alpha)} ; .\right) u_{n_{\beta}, b}\left(k^{(\beta)} ; .\right) \overline{u_{n_{\gamma}, c}}\left(k^{(\gamma)} ; .\right) \overline{u_{n_{j}, d}}\left(k^{(j)} ; .\right) \\
= & \frac{\omega_{*}}{2} \int_{U} \chi_{\mathrm{ci}}^{(3)}\left[2\left(u_{n_{\alpha}}\left(k^{(\alpha)} ; .\right) \cdot \overline{u_{n_{\gamma}}}\left(k^{(\gamma)} ; .\right)\right) u_{n_{\beta}}\left(k^{(\beta)} ; .\right)\right. \\
& \left.\quad+\left(u_{n_{\alpha}}\left(k^{(\alpha)} ; .\right) \cdot u_{n_{\beta}}\left(k^{(\beta)} ; .\right)\right) \overline{u_{n_{\gamma}}}\left(k^{(\gamma)} ; .\right)\right] \cdot \overline{u_{n_{j}}}\left(k^{(j)} ; .\right) .
\end{aligned}
$$

The symmetries in $\Gamma_{a, b, c}^{(d)}$ imply symmetries in $I_{\alpha, \beta, \gamma, j}$. Namely, due to the symmetries $\Gamma_{a, b, c}^{(d)}=\Gamma_{b, a, c}^{(d)}$ and $\Gamma_{a, b, c}^{(d)}=\Gamma_{a, b, d}^{(c)}$ we have

$$
I_{\alpha, \beta, \gamma, j}=I_{\beta, \alpha, \gamma, j} \text { and } I_{\alpha, \beta, \gamma, j}=I_{\alpha, \beta, j, \gamma} \quad \text { for all } \alpha, \beta, \gamma, j \in\{1, \ldots, N\},
$$

and due to $\Gamma_{a, b, c}^{(d)}=\Gamma_{c, d, a}^{(b)}$ we have

$$
I_{\alpha, \beta, \gamma, j}=\overline{I_{\gamma, j, \alpha, \beta}} \quad \text { for all } \alpha, \beta, \gamma, j \in\{1, \ldots, N\} .
$$

Symmetries (3.23) and (3.24) imply, in particular, that $I_{\alpha, \beta, \alpha, \beta}=I_{\alpha, \beta, \beta, \alpha} \in \mathbb{R}$ for all $\alpha, \beta \in\{1, \ldots, N\}$.

Let the crystal satisfy the rotational symmetry $\eta(x)=\eta\left(r_{\nu}(x)\right)$ and $\chi_{\mathrm{ci}}^{(3)}(x)=\chi_{\mathrm{ci}}^{(3)}\left(r_{\nu}(x)\right)$ for all $x \in \mathbb{R}^{2}$ and some $\nu \in(-\pi, \pi]$ and let $U$ be chosen so that $r_{\nu}(U)=U$. If for each $m \in\{\alpha, \beta, \gamma, j\} \subset\{1, \ldots, N\}$ there exists $m^{\prime} \in\{1, \ldots, N\}$ such that

$$
k^{\left(m^{\prime}\right)}=r_{\nu}\left(k^{(m)}\right)
$$

and if $\omega_{n}\left(k^{(m)}\right)$ is a geometrically simple eigenvalue of (2.3) for each $m \in\{\alpha, \beta, \gamma, j\}$, then

$$
I_{\alpha, \beta, \gamma, j}=I_{\alpha^{\prime}, \beta^{\prime}, \gamma^{\prime}, j^{\prime}}
$$


This is seen by the change of variables $y=r_{\nu}(x)$ in (3.22), using the facts $r_{\nu}(U)=U$ and $r_{\nu}(v) \cdot r_{\nu}(w)=v \cdot w$ for all $v, w \in \mathbb{C}^{3}$, and employing the symmetry (2.14).

Additional symmetries in $I_{\alpha, \beta, \gamma, j}$ arise when a spatial reflection symmetry in $\eta$ and $\chi_{\mathrm{ci}}^{(3)}$ is present. For instance when $\eta(x)=\eta\left(S_{2}(x)\right), \chi_{\mathrm{ci}}^{(3)}(x)=\chi_{\mathrm{ci}}^{(3)}\left(S_{2}(x)\right)$ for all $x \in \mathbb{R}^{2}$ (see Section 2.2.2) and if for each $m \in\{\alpha, \beta, \gamma, j\} \subset\{1, \ldots, N\}$ there exists $m^{\prime} \in\{1, \ldots, N\}$ such that

$$
k^{\left(m^{\prime}\right)}=S_{2}\left(k^{(m)}\right)
$$

and such that $S_{2}\left(k^{(m)}\right) \doteq k^{(m)}$ does not hold for any $m \in\{\alpha, \beta, \gamma, j\}$, then

$$
I_{\alpha, \beta, \gamma, j}=I_{\alpha^{\prime}, \beta^{\prime}, \gamma^{\prime}, j^{\prime}}
$$

This is proved via a change of variables in (3.22) using (2.21), where $a=0$ due to our assumptions. A similar result holds for the reflection symmetry in $x_{1}$.

Returning now back to (3.21), for smooth envelopes $A_{j}$ we can neglect the contribution of $\widehat{A}_{j}$ from $\ell \in \mathbb{R}^{2} \backslash D_{\varepsilon^{r-1}}$ or simply assume that $\widehat{A}_{j}$ satisfy (3.21) also there. This step can be rigorously justified via a persistence argument similar to that in [13, 14]. $\tilde{h}_{\alpha, \beta, \gamma}^{(\varepsilon)}$ will then be replaced by $\widehat{A}_{\alpha} * \widehat{A}_{\beta} * \widehat{\bar{A}}_{\gamma}$. The inverse Fourier transform then produces the coupled mode equations

$$
\Omega A_{j}+\frac{1}{2}\left(\partial_{k_{1}}^{2} \omega_{n_{j}}\left(k^{(j)}\right) \partial_{y_{1}}^{2}+\partial_{k_{2}}^{2} \omega_{n_{j}}\left(k^{(j)}\right) \partial_{y_{2}}^{2}+2 \partial_{k_{1}, k_{2}}^{2} \omega_{n_{j}}\left(k^{(j)}\right) \partial_{y_{1}, y_{2}}^{2}\right) A_{j}+\mathcal{N}_{j}=0
$$

on $\mathbb{R}^{2}$, where $\mathcal{N}_{j}$ is given by

$$
\mathcal{N}_{j}=\sum_{\substack{\alpha, \beta, \gamma \in\{1, \ldots, N\} \\ \mathcal{A}_{\alpha, \beta, \gamma, j \neq \emptyset}}} I_{\alpha, \beta, \gamma, j} A_{\alpha} A_{\beta} \overline{A_{\gamma}}
$$

Note that the coupled mode equations have the same general structure as those for gap solitons of the scalar Gross-Pitaevskii equation [13.

A localized solution $A$ of (3.27) should produce via (3.1) an approximation of a gap soliton of the Maxwell problem (1.9). A rigorous justification of this statement can be done via the Lyapunov-Schmidt reduction similarly to [12, 13, 14] and will be the subject of a future project. System (3.27) does not have localized solutions for arbitrary values of coefficients. The coefficients of the derivative terms are given by the band structure and $\Omega= \pm 1$ is determined by the condition that $\omega=\omega_{*}+\varepsilon^{2} \Omega$ lies in the gap. But the function $\chi_{\mathrm{ci}}^{(3)}$ in $I_{\alpha, \beta, \gamma, j}$ has not been fixed and remains free at this point.

The linear part of the operator in (3.27) is definite due to our assumption (A4) in Section 3.1 and the fact that $\Omega<0$ at upper edges and $\Omega>0$ at lower edges. The linear part of the operator is positive definite at lower edges $\omega_{*}$, where $k^{(j)}$ are points of maxima and negative definite at upper edges. In case $N=1$, where $\mathcal{N}_{1}=\gamma\left|A_{1}\right|^{2} A_{1}$ and $\gamma=\frac{3 \omega_{*}}{2} \int_{U} \chi_{\text {ci }}^{(3)}\left|u_{n_{j}}\left(k^{(1)} ; .\right)\right|^{4}$, a localized solution exists in the upper edge case only if $\chi_{\mathrm{ci}}^{(3)}$ is such that $\gamma>0$ while in the lower edge case $\chi_{\mathrm{ci}}^{(3)}$ has to produce $\gamma<0$. Physically it makes sense to set $\chi_{\mathrm{ci}}^{(3)}=0$ there, where $\eta=1$ (i.e. in vacuum/air). In the annulus regions, where $\eta=2.1025$, we set $\chi_{\mathrm{ci}}^{(3)}=1$ (a focusing nonlinearity) if $\gamma>0$ is needed and $\chi_{\mathrm{ci}}^{(3)}=-1$ (a defocusing nonlinearity) if $\gamma<0$ is required. This is in agreement with previous results on bifurcation of gap solitons from spectral edges in the periodic nonlinear Schrödinger equation [17, 3, 23, 12, 13, where bifurcation from upper/lower edges occurs for the focusing/defocusing nonlinearity respectively. In the case $N>1$ our numerical examples produce all $I_{\alpha, \beta, \gamma, j}$ of the same sign so that we set in the annulus regions, once again, $\chi_{\mathrm{ci}}^{(3)}=1$ if $\omega_{*}$ is an upper edge of a gap and $\chi_{\mathrm{ci}}^{(3)}=-1$ if it is a lower edge. 
3.4. Examples of Coupled Mode Equations. We present next coupled mode equations for gap solitons in the vicinity of spectral edges for the example in Section 2.3 as well as for other canonical examples. As seen in Figure 2.2, there are 3 spectral gaps $\left(0, s_{1}\right),\left(s_{2}, s_{3}\right)$ and $\left(s_{4}, s_{5}\right)$ on the positive part of the spectral $\omega$ axis for this specific example. We have the numerical values

$$
\begin{gathered}
s_{1}=\omega_{1}(\Gamma) \approx 3.610, s_{2}=\omega_{6}(\Gamma) \approx 3.701, s_{3}=\omega_{7}(\Gamma) \approx 3.750 \\
s_{4}=\omega_{12}(0,2.351) \approx 3.873, s_{5}=\omega_{13}(0,2.407) \approx 3.882 .
\end{gathered}
$$

At $s_{1}$ and $s_{2}$ several bands lie very close to each other at the extremal point $k=\Gamma$. It is, however, not known whether these truly touch and the eigenvalues have higher multiplicity than one. Numerical tests have shown that varying the value of $\eta$ for the annulus material does not change the ordering of bands at $k=\Gamma$ near $s_{1}$ and $s_{2}$. We, therefore, assume that the edges $s_{1}$ and $s_{2}$ are simple eigenvalues at $k=\Gamma$ leading to $N=1$ at $s_{1}$ and $s_{2}$. If it can be proved that, for instance, $s_{1}$ is indeed a double eigenvalue, then $N=2$ at $s_{1}$. Likewise, $N$ would change if the multiplicity could be established for $s_{2}$.

Similarly, the band $\omega_{12}$ is close to $\omega=s_{5}$ at four distinct $k$-points along $\partial \mathbb{B}_{0}$. At the point $k=(0,2.351)$ the numerical value is maximal and an analogous test shows that it remains maximal for a range of values of $\eta$. We thus assume that within $\mathbb{B}_{0}$ the value $\omega=s_{5}$ is attained only at $k=(0,2.351)$. Due to the discrete rotational symmetry of the band structure we thus have $N=6$ at $s_{5}$. Analogously, we have $N=6$ at $s_{4}$.

Except for the simplest case with $N=1$, like in Section 3.4.1 we determine the sets $\mathcal{A}_{\alpha, \beta, \gamma, j}$ using a Matlab program. First of all, it is clear that for any $k^{b} \in \mathbb{B}$ the sets $M_{b}$ and $M_{b}^{(2)}$ contain only those $(n, o, q) \in \mathbb{Z}^{2}$ with $n_{l}, o_{l}, q_{l} \in\{-1,0,1\}$ for $l=1,2$. To determine $\mathcal{A}_{\alpha, \beta, \gamma, j}$, we therefore need to test only finitely many integer vectors $(n, o, q)$ for conditions (3.12), (3.13). For an example with $N=3$ we show in Section 3.4 .2 the resulting sets $\mathcal{A}_{\alpha, \beta, \gamma, j}$ computed using this routine.

\subsubsection{Coupled Mode Equations near Edges for the Example in Section 2.3.}

Coupled Mode Equations near the Edges $s_{1}, s_{2}$ and $s_{3}(N=1)$.

At the edges $s_{1}, s_{2}$ and $s_{3}$ in Figure 2.2 the situation is particularly simple. As discussed at the beginning of Section 3.4 we have $N=1$ and $k^{(1)}=\Gamma=\left(\begin{array}{l}0 \\ 0\end{array}\right)$. Since $k^{(1)} \in \operatorname{int}(\mathbb{B})$, any (small) neighborhood of $k^{(1)}$ lies completely within $\mathbb{B}$ and thus $M_{1}=\left\{\left(\begin{array}{l}0 \\ 0\end{array}\right)\right\}$. A simple inspection determines that we have $\mathcal{A}_{1,1,1,1}=$ $\left\{\left(\left(\begin{array}{l}0 \\ 0\end{array}\right),\left(\begin{array}{l}0 \\ 0\end{array}\right),\left(\begin{array}{l}0 \\ 0\end{array}\right)\right)\right\}$. The resulting coupled mode equation for $A=A_{1}$ is

$$
\left(\Omega+\alpha\left(\partial_{y_{1}}^{2}+\partial_{y_{2}}^{2}\right)\right) A+\gamma|A|^{2} A=0,
$$

where $\alpha=\frac{1}{2} \partial_{k_{1}}^{2} \omega_{n_{1}}(\Gamma)=\frac{1}{2} \partial_{k_{2}}^{2} \omega_{n_{1}}(\Gamma)($ cf. (2.23) $)$ and $\gamma=I_{1,1,1,1}$.

The three cases $s_{1}, s_{2}$ and $s_{3}$ differ by the value of $n_{1}$, i.e. the band index. At $\omega_{*}=s_{1}$ we have $n_{1}=1$, at $\omega_{*}=s_{2}$ we have $n_{1}=6$ and at $\omega_{*}=s_{3}$ we have $n_{1}=7$. And, as discussed at the end of Section 3.3, at the upper edges $s_{1}, s_{3}$ we have $\Omega=-1$ and the function $\chi_{\mathrm{ci}}^{(3)}$ has the value 1 in the annulus regions and 0 otherwise. At $s_{2}$ we have $\Omega=1$ and $\chi_{\mathrm{ci}}^{(3)}=-1$ in the annuli.

In Section 4.1 we present a numerical example on a gap soliton approximation near $s_{2}$. We list here, therefore, the numerical values of the CME coefficients for the case $s_{2}$ :

$$
\omega_{*}=s_{2} \approx 3.701: \alpha \approx-0.0107, \gamma \approx-3.057 \text {. }
$$

Coupled Mode Equations near the Edge $s_{5}(N=6)$.

At the upper edge $s_{5}$ in Figure 2.2 we have $N=6, n_{1}=n_{2}=\ldots=n_{6}=13, k^{(1)} \approx(0,2.458)$ lying on the line from $\Gamma$ to $r_{2 \pi / 3}(M)$, and $k^{(j)}, j=2, \ldots, 6$, obtained via a rotation of $k^{(1)}$. In detail

$$
k^{(j)}=r_{(j-1) \frac{\pi}{3}}\left(k^{(1)}\right) \quad \text { for } j=2, \ldots, 6
$$


The symmetry properties (2.15), (2.19), and (2.20) produce relations among the linear coefficients of the CMEs. The sets $\mathcal{A}_{\alpha, \beta, \gamma, j}$ are either empty or contain solely the element $\left\{\left(\begin{array}{l}0 \\ 0\end{array}\right),\left(\begin{array}{l}0 \\ 0\end{array}\right),\left(\begin{array}{l}0 \\ 0\end{array}\right)\right\}$ as checked by the Matlab routine. The resulting CMEs are

$$
\begin{aligned}
\left(\Omega+\alpha_{1} \partial_{y_{1}}^{2}+\beta_{1} \partial_{y_{2}}^{2}\right) A_{1}+N_{1}= & 0, \\
\left(\Omega+\alpha_{2} \partial_{y_{1}}^{2}+\beta_{2} \partial_{y_{2}}^{2}+\mu \partial_{y_{1}, y_{2}}^{2}\right) A_{2}+N_{2}= & 0, \\
\left(\Omega+\alpha_{2} \partial_{y_{1}}^{2}+\beta_{2} \partial_{y_{2}}^{2}-\mu \partial_{y_{1}, y_{2}}^{2}\right) A_{3}+N_{3}= & 0, \\
\left(\Omega+\alpha_{1} \partial_{y_{1}}^{2}+\beta_{1} \partial_{y_{2}}^{2}\right) A_{4}+N_{4}= & 0, \\
\left(\Omega+\alpha_{2} \partial_{y_{1}}^{2}+\beta_{2} \partial_{y_{2}}^{2}+\mu \partial_{y_{1}, y_{2}}^{2}\right) A_{5}+N_{5}= & 0, \\
\left(\Omega+\alpha_{2} \partial_{y_{1}}^{2}+\beta_{2} \partial_{y_{2}}^{2}-\mu \partial_{y_{1}, y_{2}}^{2}\right) A_{6}+N_{6}= & 0,
\end{aligned}
$$

where $\Omega=-1, \alpha_{1}=\partial_{k_{1}}^{2} \omega_{13}\left(k^{(1)}\right), \beta_{1}=\partial_{k_{2}}^{2} \omega_{13}\left(k^{(1)}\right), \alpha_{2}=\frac{1}{4}\left(\alpha_{1}+3 \beta_{1}\right), \beta_{2}=\frac{1}{4}\left(3 \alpha_{1}+\beta_{1}\right), \mu=\frac{\sqrt{3}}{4}\left(\alpha_{1}-\beta_{1}\right)=$ $\partial_{k_{1}, k_{2}}^{2} \omega_{13}\left(k^{(2)}\right)$, and

$$
\begin{aligned}
& N_{1}=2 \sum_{i=1}^{6} I_{i, 1, i, 1}\left|A_{i}\right|^{2} A_{1}-I_{1,1,1,1}\left|A_{1}\right|^{2} A_{1}+2\left(I_{2,5,4,1} A_{2} A_{5}+I_{3,6,4,1} A_{3} A_{6}\right) \bar{A}_{4} \\
& N_{2}=2 \sum_{i=1}^{6} I_{i, 2, i, 2}\left|A_{i}\right|^{2} A_{2}-I_{2,2,2,2}\left|A_{2}\right|^{2} A_{2}+2\left(I_{1,4,5,2} A_{1} A_{4}+I_{3,6,5,2} A_{3} A_{6}\right) \bar{A}_{5} \\
& N_{3}=2 \sum_{i=1}^{6} I_{i, 3, i, 3}\left|A_{i}\right|^{2} A_{3}-I_{3,3,3,3}\left|A_{3}\right|^{2} A_{3}+2\left(I_{1,4,6,3} A_{1} A_{4}+I_{2,5,6,3} A_{2} A_{5}\right) \bar{A}_{6} \\
& N_{4}=2 \sum_{i=1}^{6} I_{i, 4, i, 4}\left|A_{i}\right|^{2} A_{4}-I_{4,4,4,4}\left|A_{4}\right|^{2} A_{4}+2\left(I_{2,5,1,4} A_{2} A_{5}+I_{3,6,1,4} A_{3} A_{6}\right) \bar{A}_{1} \\
& N_{5}=2 \sum_{i=1}^{6} I_{i, 5, i, 5}\left|A_{i}\right|^{2} A_{5}-I_{5,5,5,5}\left|A_{5}\right|^{2} A_{5}+2\left(I_{1,4,2,5} A_{1} A_{4}+I_{3,6,2,5} A_{3} A_{6}\right) \bar{A}_{2} \\
& N_{6}=2 \sum_{i=1}^{6} I_{i, 6, i, 6}\left|A_{i}\right|^{2} A_{6}-I_{6,6,6,6}\left|A_{6}\right|^{2} A_{6}+2\left(I_{1,4,3,6} A_{1} A_{4}+I_{2,5,3,6} A_{2} A_{5}\right) \bar{A}_{3} .
\end{aligned}
$$

Due to symmetries, many of the coefficients in the nonlinear terms are equal. Symmetry (3.25) with $\nu=\pi / 3$ and symmetry (3.23) imply

$$
\begin{aligned}
\gamma_{0} & :=I_{1,1,1,1}=I_{2,2,2,2}=\ldots=I_{6,6,6,6}, \\
\gamma_{1} & :=I_{2,1,2,1}=I_{3,2,3,2}=\ldots=I_{6,5,6,5}=I_{1,6,1,6} \\
& =I_{1,2,1,2}=I_{2,3,2,3}=\ldots=I_{5,6,5,6}=I_{6,1,6,1}, \\
\gamma_{2} & :=I_{3,1,3,1}=I_{4,2,4,2}=I_{5,3,5,3}=I_{6,4,6,4}=I_{1,5,1,5}=I_{2,6,2,6} \\
& =I_{1,3,1,3}=I_{2,4,2,4}=I_{3,5,3,5}=I_{4,6,4,6}=I_{5,1,5,1}=I_{6,2,6,2}, \\
\gamma_{3} & :=I_{4,1,4,1}=I_{5,2,5,2}=I_{6,3,6,3}=I_{1,4,1,4}=I_{2,5,2,5}=I_{3,6,3,6}, \\
\gamma_{4} & :=I_{2,5,1,4}=I_{3,6,2,5}=I_{1,4,3,6}=I_{2,5,4,1}=I_{3,6,5,2}=I_{1,4,6,3} .
\end{aligned}
$$

Using (3.24) and (3.23), we get

$$
\overline{\gamma_{4}}=I_{3,6,4,1}=I_{3,6,1,4}=I_{1,4,5,2}=I_{1,4,2,5}=I_{2,5,6,3}=I_{2,5,3,6} .
$$

We have $\gamma_{0}, \gamma_{1}, \gamma_{2}, \gamma_{3} \in \mathbb{R}$ as explained below (3.24). 
Finally, because $k^{(1)}=\left(k_{1}^{(4)},-k_{2}^{(4)}\right)^{T}, k^{(2)}=\left(k_{1}^{(3)},-k_{2}^{(3)}\right)^{T}$, and $k^{(5)}=\left(k_{1}^{(6)},-k_{2}^{(6)}\right)^{T}$ with $k^{(1)}, k^{(2)}$ and $k^{(5)}$ lying in the interior of $\mathbb{B}$ away from the line $k_{2}=0$, the symmetry (3.26) applies and we get

$$
I_{2,5,4,1}=I_{3,6,1,4} \text {. }
$$

Therefore

$$
I_{2,5,4,1}=I_{3,6,1,4}=I_{3,6,4,1}=I_{4,1,5,2}=\overline{I_{2,5,4,1}}
$$

so that also $\gamma_{4} \in \mathbb{R}$. The second, third and fourth equalities in (3.30) hold due to (3.23), (3.25), and (3.24).

As a result the nonlinear terms in (3.29) can be simplified to

$$
\begin{aligned}
& N_{1}:=2\left(\frac{\gamma_{0}}{2}\left|A_{1}\right|^{2}+\gamma_{1}\left(\left|A_{2}\right|^{2}+\left|A_{6}\right|^{2}\right)+\gamma_{2}\left(\left|A_{3}\right|^{2}+\left|A_{5}\right|^{2}\right)+\gamma_{3}\left|A_{4}\right|^{2}\right) A_{1}+2 \gamma_{4}\left(A_{2} A_{5}+A_{3} A_{6}\right) \overline{A_{4}}, \\
& N_{2}:=2\left(\frac{\gamma_{0}}{2}\left|A_{2}\right|^{2}+\gamma_{1}\left(\left|A_{1}\right|^{2}+\left|A_{3}\right|^{2}\right)+\gamma_{2}\left(\left|A_{4}\right|^{2}+\left|A_{6}\right|^{2}\right)+\gamma_{3}\left|A_{5}\right|^{2}\right) A_{2}+2 \gamma_{4}\left(A_{1} A_{4}+A_{3} A_{6}\right) \overline{A_{5}}, \\
& N_{3}:=2\left(\frac{\gamma_{0}}{2}\left|A_{3}\right|^{2}+\gamma_{1}\left(\left|A_{2}\right|^{2}+\left|A_{4}\right|^{2}\right)+\gamma_{2}\left(\left|A_{1}\right|^{2}+\left|A_{5}\right|^{2}\right)+\gamma_{3}\left|A_{6}\right|^{2}\right) A_{3}+2 \gamma_{4}\left(A_{1} A_{4}+A_{2} A_{5}\right) \overline{A_{6}}, \\
& N_{4}:=2\left(\frac{\gamma_{0}}{2}\left|A_{4}\right|^{2}+\gamma_{1}\left(\left|A_{3}\right|^{2}+\left|A_{5}\right|^{2}\right)+\gamma_{2}\left(\left|A_{2}\right|^{2}+\left|A_{6}\right|^{2}\right)+\gamma_{3}\left|A_{1}\right|^{2}\right) A_{4}+2 \gamma_{4}\left(A_{2} A_{5}+A_{3} A_{6}\right) \overline{A_{1}}, \\
& N_{5}:=2\left(\frac{\gamma_{0}}{2}\left|A_{5}\right|^{2}+\gamma_{1}\left(\left|A_{4}\right|^{2}+\left|A_{6}\right|^{2}\right)+\gamma_{2}\left(\left|A_{1}\right|^{2}+\left|A_{3}\right|^{2}\right)+\gamma_{3}\left|A_{2}\right|^{2}\right) A_{5}+2 \gamma_{4}\left(A_{1} A_{4}+A_{3} A_{6}\right) \overline{A_{2}}, \\
& N_{6}:=2\left(\frac{\gamma_{0}}{2}\left|A_{6}\right|^{2}+\gamma_{1}\left(\left|A_{1}\right|^{2}+\left|A_{5}\right|^{2}\right)+\gamma_{2}\left(\left|A_{2}\right|^{2}+\left|A_{4}\right|^{2}\right)+\gamma_{3}\left|A_{3}\right|^{2}\right) A_{6}+2 \gamma_{4}\left(A_{1} A_{4}+A_{2} A_{5}\right) \overline{A_{3}}
\end{aligned}
$$

with $\gamma_{0}, \gamma_{1}, \gamma_{2}, \gamma_{3}, \gamma_{4} \in \mathbb{R}$. A system of six CMEs with the same structure as above arises also at the edge $s_{4}$.

In Section 4.2 a numerical example of gap soliton asymptotics near $s_{5}$ is given. The numerical values of the coefficients in the CMEs (3.29) for $s_{5}$ are

$$
\begin{aligned}
\omega_{*}=s_{5} \approx 3.882: \alpha_{1} & \approx 0.0189, \alpha_{2} \approx 0.146, \beta_{1} \approx 0.189, \beta_{2} \approx 0.0614, \mu \approx-0.0736, \\
\gamma_{0} & \approx 1.282, \gamma_{1} \approx 0.789, \gamma_{2} \approx 0.757, \gamma_{3} \approx 1.193, \gamma_{4} \approx 0.714 .
\end{aligned}
$$

As $s_{5}$ is an upper edge edge, the coefficients $\gamma_{j}, j \in\{0, \ldots, 4\}$, were computed using $\chi_{\mathrm{ci}}^{(3)}=1$ in the annulus regions.

\subsubsection{Additional CME Examples.}

Example of Coupled Mode Equations for $N=2$.

An example of a situation for $N=2$ is when the locations of the extrema are $k^{(1)}=K, k^{(2)}=r_{\pi / 3}(K)$. With $b^{(1)}, b^{(2)}$ as in Section 2.3 we then have $k^{(1)}=\frac{4 \pi}{3 a_{0}}\left(\begin{array}{c}1 \\ 0\end{array}\right)$ and $k^{(2)}=\frac{2 \pi}{3 a_{0}}\left(\begin{array}{c}1 \\ \sqrt{3}\end{array}\right)$. The corresponding integer shift sets are $M_{1}=\left\{\left(\begin{array}{l}0 \\ 0\end{array}\right),\left(\begin{array}{l}0 \\ 1\end{array}\right),\left(\begin{array}{l}1 \\ 1\end{array}\right)\right\}, M_{2}=\left\{\left(\begin{array}{l}0 \\ 0\end{array}\right),\left(\begin{array}{l}1 \\ 0\end{array}\right),\left(\begin{array}{l}1 \\ 1\end{array}\right)\right\}$. Due to the rotation symmetry of the bands and their labeling according to size, we necessarily have $n_{1}=n_{2}$. We define $n_{*}:=n_{1}=n_{2}$. From (2.24) we have

$$
\partial_{k_{1}, k_{2}}^{2} \omega_{n_{*}}\left(k^{(1)}\right)=0
$$

and using (2.15) with $\alpha=\pi / 3$, we obtain

$$
\begin{aligned}
\partial_{k_{1}}^{2} \omega_{n_{*}}\left(k^{(2)}\right) & =\frac{1}{4}\left(\partial_{k_{1}}^{2} \omega_{n_{*}}\left(k^{(1)}\right)+3 \partial_{k_{2}}^{2} \omega_{n_{*}}\left(k^{(1)}\right)\right), \\
\partial_{k_{2}}^{2} \omega_{n_{*}}\left(k^{(2)}\right) & =\frac{1}{4}\left(3 \partial_{k_{1}}^{2} \omega_{n_{*}}\left(k^{(1)}\right)+\partial_{k_{2}}^{2} \omega_{n_{*}}\left(k^{(1)}\right)\right), \\
\partial_{k_{1}, k_{2}}^{2} \omega_{n_{*}}\left(k^{(2)}\right) & =\frac{\sqrt{3}}{4}\left(\partial_{k_{1}}^{2} \omega_{n_{*}}\left(k^{(1)}\right)-\partial_{k_{2}}^{2} \omega_{n_{*}}\left(k^{(1)}\right)\right) .
\end{aligned}
$$

After having numerically checked the sets $\mathcal{A}_{\alpha, \beta, \gamma, j}$ for all combinations of $\alpha, \beta, \gamma, j$ to determine the nonlinear terms, we thus arrive at the CMEs

$$
\begin{array}{r}
\left(\Omega+\alpha_{1} \partial_{y_{1}}^{2}+\beta_{1} \partial_{y_{2}}^{2}\right) A_{1}+\left(\gamma_{0}\left|A_{1}\right|^{2}+2 \gamma_{1}\left|A_{2}\right|^{2}\right) A_{1}=0 \\
\left(\Omega+\alpha_{2} \partial_{y_{1}}^{2}+\beta_{2} \partial_{y_{2}}^{2}+\mu \partial_{y_{1}, y_{2}}^{2}\right) A_{2}+\left(\gamma_{0}\left|A_{2}\right|^{2}+2 \gamma_{1}\left|A_{1}\right|^{2}\right) A_{2}=0
\end{array}
$$


where $\alpha_{1}=\frac{1}{2} \partial_{k_{1}}^{2} \omega_{n_{*}}\left(k^{(1)}\right), \beta_{1}=\frac{1}{2} \partial_{k_{2}}^{2} \omega_{n_{*}}\left(k^{(1)}\right)$, and $\alpha_{2}=\frac{1}{4}\left(\alpha_{1}+3 \beta_{1}\right), \beta_{2}=\frac{1}{4}\left(3 \alpha_{1}+\beta_{1}\right), \mu=\frac{\sqrt{3}}{2}\left(\alpha_{1}-\beta_{1}\right)$, $\gamma_{0}:=I_{1,1,1,1}=I_{2,2,2,2}$ using symmetry (3.25) with $\nu=\pi / 3$, and $\gamma_{1}:=I_{1,2,1,2}=I_{1,2,2,1}$ using (3.23).

Example of Coupled Mode Equations for $N=3$..

Let us assume that a gap edge for $N=3$ has extremal points at $k^{(1)}=M, k^{(2)}=r_{\pi / 3}(M), k^{(3)}=$ $r_{2 \pi / 3}(M)$. With the choice of the reciprocal lattice vectors $b^{(1)}, b^{(2)}$ as in Section 2.3 we have $k^{(1)}=\frac{1}{2} b^{(2)}$, $k^{(2)}=\frac{1}{2}\left(b^{(1)}+b^{(2)}\right)$, and $k^{(3)}=\frac{1}{2} b^{(1)}$ with the corresponding integer shift sets $M_{1}=\left\{\left(\begin{array}{l}0 \\ 0\end{array}\right),\left(\begin{array}{l}0 \\ 1\end{array}\right)\right\}, M_{2}=$ $\left\{\left(\begin{array}{l}0 \\ 0\end{array}\right),\left(\begin{array}{l}1 \\ 1\end{array}\right)\right\}$, and $M_{3}=\left\{\left(\begin{array}{l}0 \\ 0\end{array}\right),\left(\begin{array}{l}1 \\ 0\end{array}\right)\right\}$. Similarly to Section 3.4.2 we have $n_{1}=n_{2}=n_{3}=: n_{*}$. Using (2.15) and (2.25) -(2.27), we get

$$
\begin{aligned}
& \partial_{k_{1}}^{2} \omega_{n_{*}}\left(k^{(1)}\right)=\partial_{k_{1}}^{2} \omega_{n_{*}}\left(k^{(2)}\right)=\partial_{k_{1}}^{2} \omega_{n_{*}}\left(k^{(3)}\right)=\partial_{k_{2}}^{2} \omega_{n_{*}}\left(k^{(1)}\right)=\partial_{k_{2}}^{2} \omega_{n_{*}}\left(k^{(2)}\right)=\partial_{k_{2}}^{2} \omega_{n_{*}}\left(k^{(3)}\right)=: \alpha, \\
& \partial_{k_{1}, k_{2}}^{2} \omega_{n_{*}}\left(k^{(1)}\right)=\partial_{k_{1}, k_{2}}^{2} \omega_{n_{*}}\left(k^{(2)}\right)=\partial_{k_{1}, k_{2}}^{2} \omega_{n_{*}}\left(k^{(3)}\right)=0 .
\end{aligned}
$$

The sets $\mathcal{A}_{\alpha, \beta, \gamma, j}$ are, once again, determined using the Matlab routine and the results are for illustration

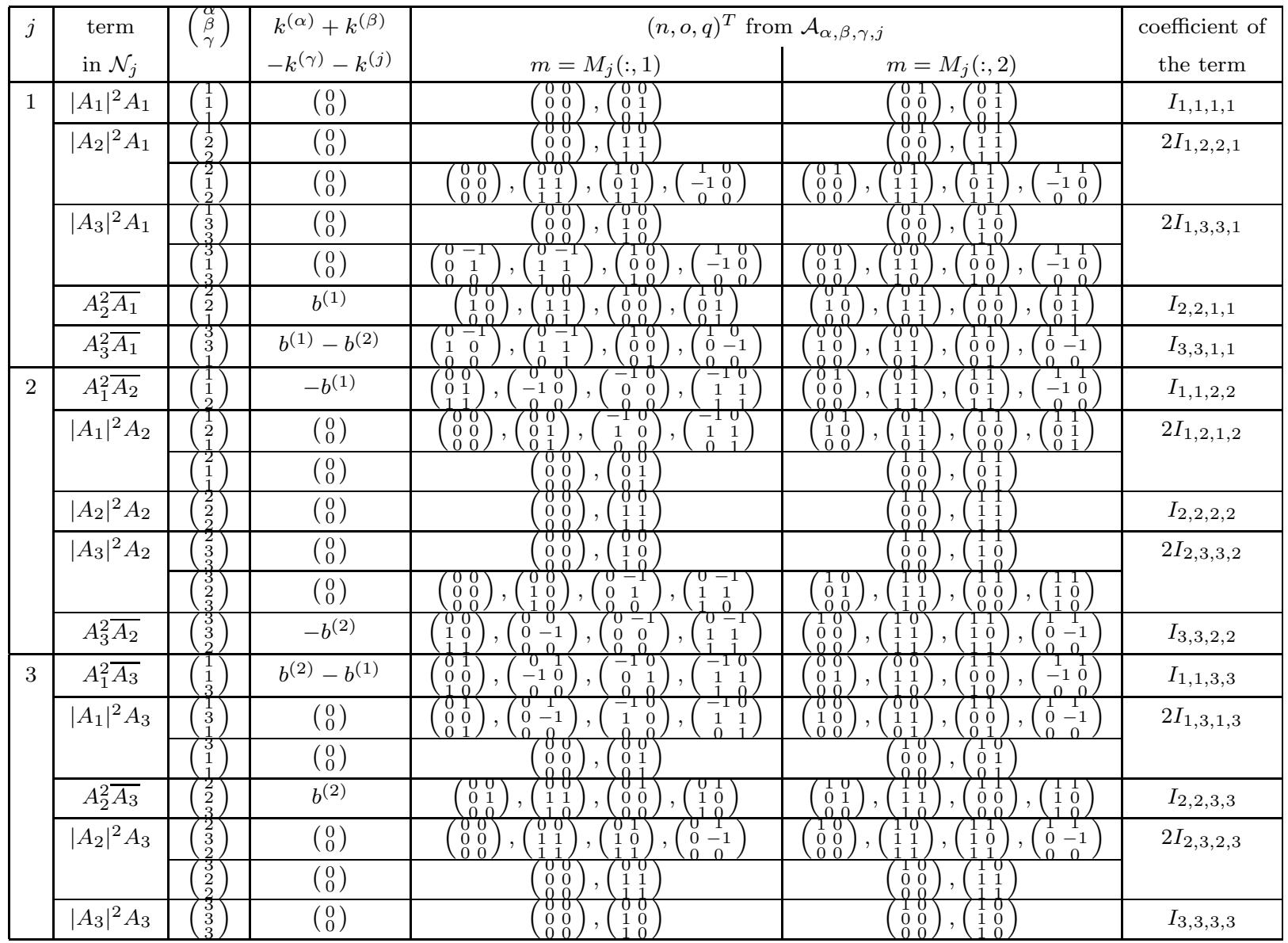

Table 3.1: Calculation of the nonlinear terms for Section 3.4 .2

listed in Table 3.1. The resulting CMEs are

$$
\begin{aligned}
& \left(\Omega+\alpha\left(\partial_{y_{1}}^{2}+\partial_{y_{2}}^{2}\right)\right) A_{1}+\left(\gamma_{0}\left|A_{1}\right|^{2}+2 \gamma_{1}\left(\left|A_{2}\right|^{2}+\left|A_{3}\right|^{2}\right)\right) A_{1}+\gamma_{2}\left(A_{2}^{2}+A_{3}^{2}\right) \overline{A_{1}}=0 \\
& \left(\Omega+\alpha\left(\partial_{y_{1}}^{2}+\partial_{y_{2}}^{2}\right)\right) A_{2}+\left(\gamma_{0}\left|A_{2}\right|^{2}+2 \gamma_{1}\left(\left|A_{1}\right|^{2}+\left|A_{3}\right|^{2}\right)\right) A_{2}+\gamma_{2}\left(A_{1}^{2}+A_{3}^{2}\right) \overline{A_{2}}=0 \\
& \left(\Omega+\alpha\left(\partial_{y_{1}}^{2}+\partial_{y_{2}}^{2}\right)\right) A_{3}+\left(\gamma_{0}\left|A_{3}\right|^{2}+2 \gamma_{1}\left(\left|A_{1}\right|^{2}+\left|A_{2}\right|^{2}\right)\right) A_{3}+\gamma_{2}\left(A_{1}^{2}+A_{2}^{2}\right) \overline{A_{3}}=0
\end{aligned}
$$


where the following symmetries have been used: $\gamma_{0}:=I_{1,1,1,1}=I_{2,2,2,2}=I_{3,3,3,3}$ due to (3.25) with $\nu=\pi / 3$; $\gamma_{1}:=I_{1,2,2,1}=I_{2,3,3,2}=I_{1,2,1,2}=I_{2,3,2,3}$ due to (3.25) with $\nu=\pi / 3$, and (3.23). Moreover, $\gamma_{1}=I_{2,1,1,2}=$ $I_{1,3,3,1}=I_{3,1,1,3}$, where the second equality follows from (3.25) with $\nu=\pi / 3$ and the facts that $k^{(1)}=$ $r_{\pi / 3}\left(k^{(3)}-b^{(1)}\right)$ and $u_{n}\left(k^{(3)}-b^{(1)} ; x\right)=u_{n}\left(k^{(3)} ; x\right)$ for all $n \in \mathbb{N}$. Finally $\gamma_{2}:=I_{2,2,1,1}=I_{3,3,2,2}=\overline{I_{1,1,2,2}}=$ $\overline{I_{2,2,3,3}}$ due to (3.25) and (3.24), and $\gamma_{2}=I_{2,2,1,1}=I_{1,1,3,3}$ using (3.25) together with $k^{(1)}=r_{\pi / 3}\left(k^{(3)}-b^{(1)}\right.$ ) and $u_{n}\left(k^{(3)}-b^{(1)} ; x\right)=u_{n}\left(k^{(3)} ; x\right)$ for all $n \in \mathbb{N}$. All the nonlinear coefficients are real: $\gamma_{0}, \gamma_{1} \in \mathbb{R}$ due to (3.24) and $\gamma_{2} \in \mathbb{R}$ since $\gamma_{2}=I_{2,2,1,1}=\overline{I_{1,1,2,2}}$ by (3.24) and at the same time $\gamma_{2}=I_{2,2,1,1}=I_{1,1,2,2}$ by (3.26), where we are using the facts that $k^{(2)}=\left(k_{1}^{(1)},-k_{2}^{(1)}\right)^{T}$ and that $k^{(2)} \doteq k^{(1)}$ does not hold.

4. Numerical Examples of Gap Soliton Approximations. We compute here numerically localized solutions of the CMEs for the examples $s_{2}, s_{5}$ in Section 3.4.1. Then, using the leading order term in (3.1), we generate and plot an approximation of a gap soliton of the nonlinear Maxwell problem (1.9). In the evaluation of (3.1) we position the photonic crystal so that the center of one of the annuli lies at the origin $x=0$.

4.1. Gap Soliton near the Edge $s_{2}$. Figure 4.1 plots in (a) the unique positive localized solution, the so called Townes soliton, of (3.28) for the case $\omega_{*}=s_{2}$ and in (b) the intensity $I=\left|E_{1}\right|^{2}+\left|E_{2}\right|^{2}+\left|E_{3}\right|^{2}$ of the leading order term in (3.1). In Figure 4.2 we show the absolute value of the individual components $E_{1}, E_{2}, E_{3}$. As the Townes soliton is radially symmetric, it was computed using the shooting method on (3.28) in polar coordinates. The fourth to fifth order explicit Runge-Kutta method ODE45 of Matlab was used in the shooting method.

(a) $\mathrm{A}(\mathrm{y})$

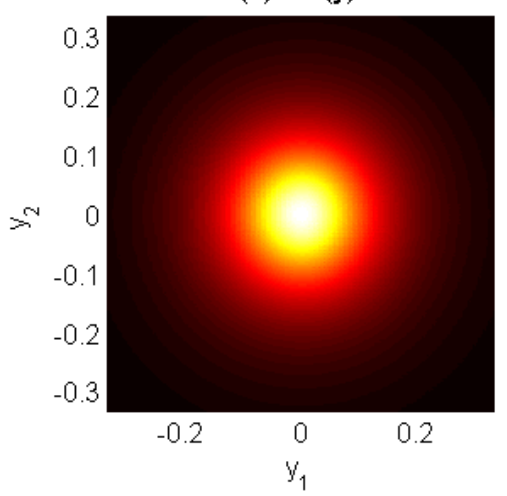

(b) $\left|E_{1}\right|^{2}+\left|E_{2}\right|^{2}+\left|E_{3}\right|^{2}$

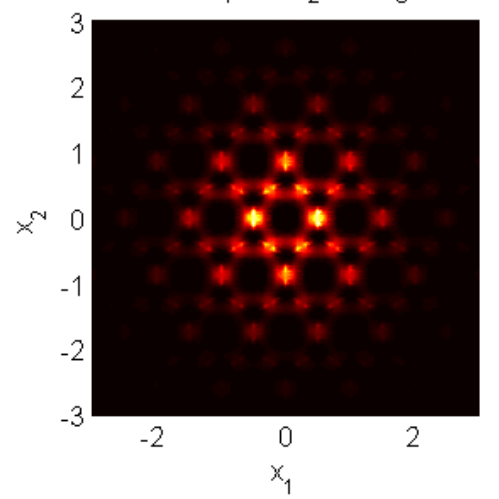

Fig. 4.1: (a) CME solution, (b) intensity of the gap soliton approximation for the case $\omega_{*}=s_{2}$. See Section 4.1 .

4.2. Gap Soliton near the Edge $s_{5}$. Here we restrict to solutions of (3.29) with the symmetry

$$
A_{1}=A_{4}, A_{2}=A_{5}, A_{3}=A_{6},
$$

which reduces the problem to a system of three equations for $A_{1}, A_{2}, A_{3}$. To find a localized solution, we first replace $\mu$ by 0 , and $\alpha_{1}, \alpha_{2}, \beta_{1}, \beta_{2}$ by the average of these four numbers. Also the coefficients in each $\mathcal{N}_{j}$, $j \in\{1,2,3\}$, are replaced by their average. For this modified system the Townes soliton with $A_{1}=A_{2}=A_{3}$ is computed via the shooting method as in Section 4.1. Then a numerical homotopy in the coefficients is used to get a solution of (3.29). The homotopy is applied to a fourth order centered finite difference discretization of (3.29). Our homotopy always results in $A_{1}=0$ so that in the end we produce a solution of (3.29) with $A_{1}=A_{4}=0$ and $A_{2}=A_{5} \neq 0, A_{3}=A_{6} \neq 0$. The two components $A_{2}, A_{3}$ are plotted in Figure 4.3 together 
(a) $\left|E_{1}\right|$

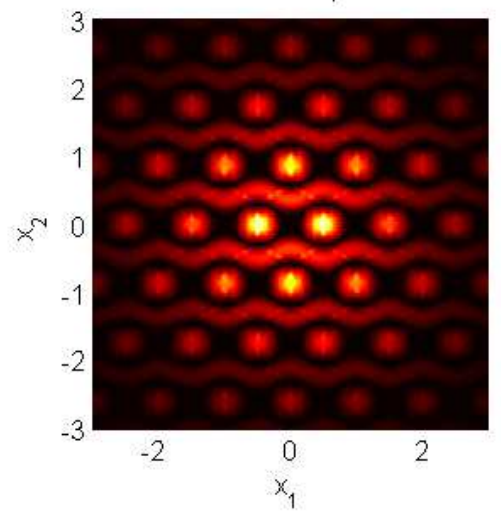

(b) $\left|E_{2}\right|$

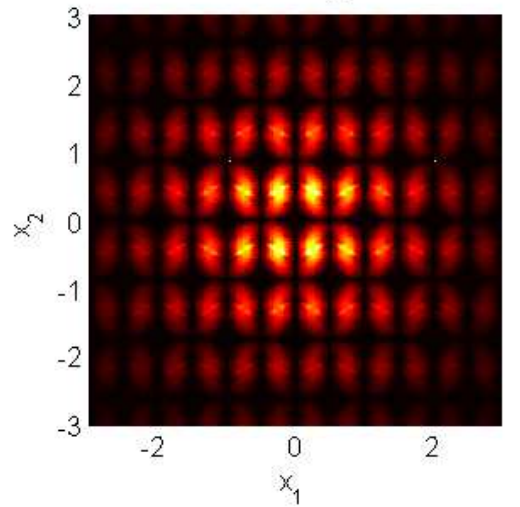

(c) $\left|E_{3}\right|$

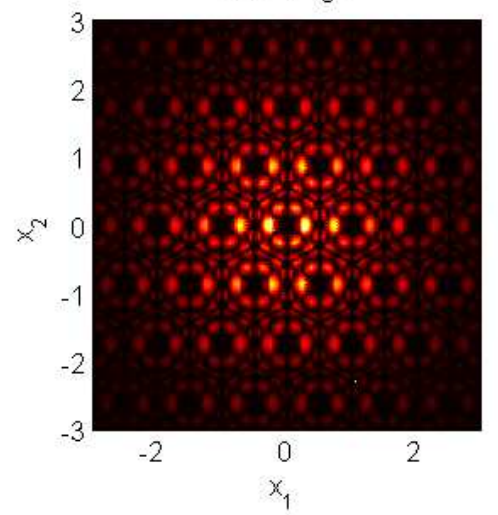

Fig. 4.2: Absolute value of the components $E_{1}, E_{2}, E_{3}$ of the gap soliton approximation for $\omega_{*}=s_{2}$. See Section 4.1

with the intensity of the corresponding leading order term in (3.1). In Figure 4.4 we plot the individual components of $E$ in absolute value.
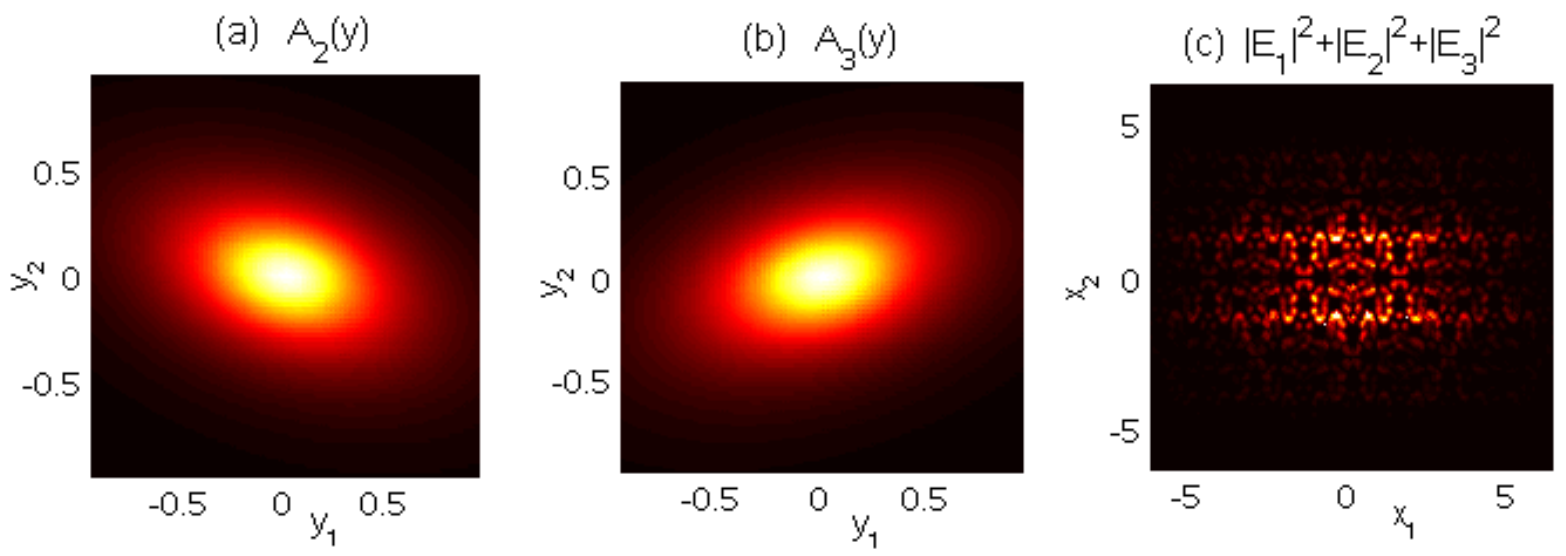

Fig. 4.3: (a) CME solutions $A_{2}, A_{3}$, (b) intensity of the gap soliton approximation for the case $\omega_{*}=s_{5}$. See Section 4.2

5. Conclusions. We have considered monochromatic out-of-plane gap solitons in Kerr nonlinear $2 \mathrm{D}$ photonic crystals as described by the full vector Maxwell system. Using a model of the nonlinear polarization which does not produce higher harmonics, we arrive at a cubically nonlinear curl-curl problem for the fundamental harmonic. For gap solitons with frequencies in spectral gaps but in an asymptotic vicinity of a gap edge we assume a standard slowly varying envelope approximation based on the gap edge Bloch waves modulated by slowly varying envelopes of small amplitude. These envelopes are then shown to satisfy a system of coupled mode equations (CMEs) of the same structure as in the case of gap solitons of the 2D periodic nonlinear Schrödinger equation [13, 14. In particular the system generally involves mixed derivatives. Being a constant coefficient system depending only on the slow variables, the CMEs is a simple effective model for the near edge gap solitons. Similarly to 13 the derivation of CMEs needs to be carried out in Bloch variables due to the possible quasi-periodicity of gap edge Bloch waves. Symmetries among the coefficients of the CMEs 
(a) $\left|E_{1}\right|$

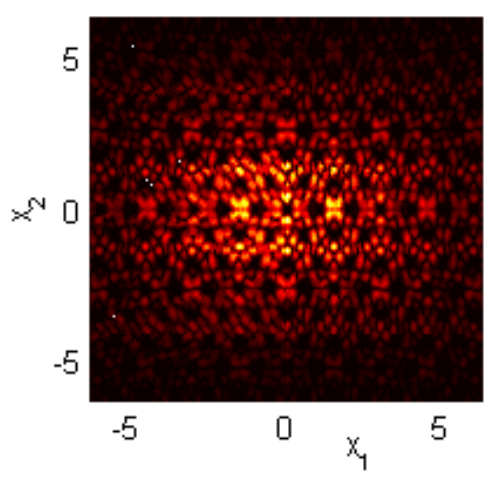

(b) $\left|E_{2}\right|$

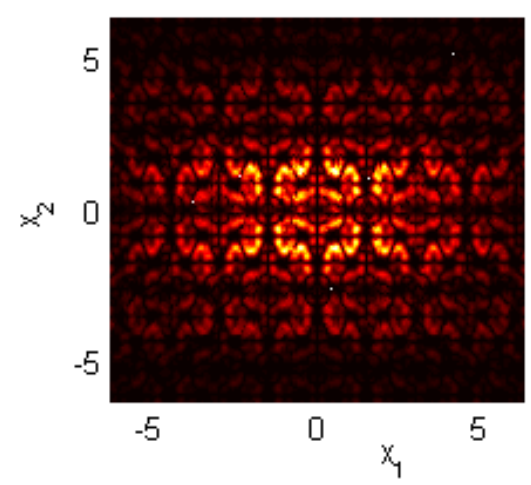

(c) $\left|E_{3}\right|$

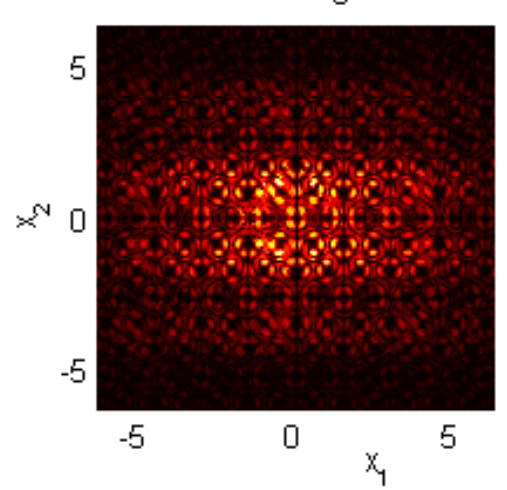

Fig. 4.4: Absolute value of the components $E_{1}, E_{2}, E_{3}$ of the gap soliton approximation for $\omega_{*}=s_{2}$. See Section 4.2

are determined using symmetries of the band structure and among the Bloch waves.

We provide an example of a photonic crystal with a hexagonal periodicity lattice and a circular material structure in the periodicity cell. For this crystal three gaps are numerically observed (for $\omega>0$ ). CMEs are then derived for several gap edges including a case where a system of six CMEs arises. Numerical computations of localized solutions of these CMEs and of the corresponding gap soliton approximations are then performed. For the CME system with six components only solutions with four nonzero components were numerically constructed and it is unclear whether a solution with all six nonzero components exists.

A rigorous justification of the CMEs, which states that for a certain class of CME solutions the full Maxwell system has gap soliton solutions which are indeed approximated by the slowly varying envelope asymptotic expansion, is expected to hold by similar arguments to those in [12, 13, 14. It will be the subject of future work.

Acknowledgments. We thank Stefan Findeisen, Karlsruhe Institute of Technology, for carrying out the finite element computations in Section 2.3, T. Dohnal was partially supported by DFG Research Training Group 1924: Analysis, Simulation and Design of Nanotechnological Processes.

\section{REFERENCES}

[1] D. Agueev and D. E. Pelinovsky. Modeling of wave resonances in low-contrast photonic crystals. SIAM J. Appl. Math., 65(4):1101-1129 (electronic), 2005.

[2] N. Aközbek and S. John. Optical solitary waves in two- and three-dimensional nonlinear photonic band-gap structures. Phys. Rev. E, 57:2287-2319, 1998.

[3] S. Alama and Y. Li. Existence of solutions for semilinear elliptic equations with indefinite linear part. J. Diff. Eq., 96(1):89-115, 1992.

[4] J. Arriaga and B. Meneses. Band structure for the cladding of a hollow core photonic crystal fibre. Rev. Mexicana Fis., 49:335-337, 2003.

[5] N. W. Ashcroft and D. N. Mermin. Solid State Physics. Thomson Learning, Toronto, 1st edition, 1976.

[6] B. Babin and A. Figotin. Nonlinear photonic crystals: IV. Nonlinear Schrödinger equation regime. Waves Rand. Med., 15:145-228, 2005.

[7] W. Bangerth, R. Hartmann, and G. Kanschat. deal.II - A general-purpose object-oriented finite element library. ACM Trans. Math. Softw., 33, 2007.

[8] G. Bao, L. Cowsar, and W. Masters. Mathematical modeling in optical science. Frontiers in applied mathematics. Society for Industrial and Applied Mathematics, Philadelphia, PA, 2001.

[9] N. A. R. Bhat and J. E. Sipe. Optical pulse propagation in nonlinear photonic crystals. Phys. Rev. E, 64:056604, 2001. 
[10] K. Busch, G. Schneider, L. Tkeshelashvili, and H. Uecker. Justification of the nonlinear Schrödinger equation in spatially periodic media. Z. Angew. Math. Phys., 57:905-939, 2006.

[11] T. Dohnal and A. B. Aceves. Optical soliton bullets in (2+1)D nonlinear Bragg resonant periodic geometries. In J. Yang, editor, Nonlinear Wave Phenomena in Periodic Photonic Structures, volume 115 of Studies Appl. Math., pages 209-232. John Wiley, Malden, USA, 2005.

[12] T. Dohnal, D.E. Pelinovsky, and G. Schneider. Coupled-mode equations and gap solitons in a two-dimensional nonlinear elliptic problem with a separable periodic potential. J. Nonlin. Sci., 19:95-131, 2009.

[13] T. Dohnal and H. Uecker. Coupled mode equations and gap solitons for the 2d Gross-Pitaevskii equation with a non-separable periodic potential. Physica D, 238(9-10):860-879, 2009.

[14] T. Dohnal and H. Uecker. Erratum to "Coupled Mode Equations and Gap Solitons for the 2D Gross-Pitaevskii Equation with a Non-separable Periodic Potential" by T. Dohnal and H. Uecker [Physica D 238 (2009), 860-879]. Physica D, 240:357-362, 2011.

[15] W. Dörfler, A. Lechleiter, M. Plum, G. Schneider, and C. Wieners. Photonic Crystals: Mathematical Analysis and Numerical Approximation. Oberwolfach Seminars. Birkhäuser Verlag, Basel, 2011.

[16] R. H. Goodman, M. I. Weinstein, and P. J. Holmes. Nonlinear propagation of light in one-dimensional periodic structures. J. Nonlin. Sci., 11(2):123-168, 2001.

[17] H. P. Heinz and T. Küpper and C. A. Stuart. Existence and bifurcation of solutions for nonlinear perturbations of the periodic Schrödinger equation. J. Diff. Eq., 100(2):341-354, 1992.

[18] T. Katō. Perturbation theory for linear operators. Grundlehren der mathematischen Wissenschaften. Springer, Berlin, 1995.

[19] P. Kuchment. Floquet theory for partial differential equations. Operator theory. Birkhäuser Verlag, Basel, 1993.

[20] P. A. Markowich and F. Poupaud. The Maxwell equation in a periodic medium: Homogenization of the energy density. Ann. Scuola Norm. Sup. Pisa, 23(2):301-324, 1996.

[21] J. V. Moloney and A. C. Newell. Nonlinear Optics. Westview Press, Oxford, 2004.

[22] P. Monk. Finite Element Methods for Maxwell's Equations. Clarendon Press, Oxford, 2003.

[23] A. Pankov. Periodic nonlinear Schrödinger equation with application to photonic crystals. Milan J. Math., 73:259-287, 2005.

[24] D. E. Pelinovsky and G. Schneider. Justification of the coupled-mode approximation for a nonlinear elliptic problem with a periodic potential. Applicable Analysis, 86:1017-1036, 2007.

[25] D. E. Pelinovsky, G. Simpson, and M. I. Weinstein. Broad band solitons in a periodic and nonlinear Maxwell system, 2011. arXiv:1106.3672v1.

[26] C. A. Stuart. Guidance properties of nonlinear planar waveguides. Arch. Ration. Mech. Anal., 125(2):145-200, 1993.

[27] R. L. Sutherland, D. G. McLean, and S. Kirkpatrick. Handbook of nonlinear optics. Optical engineering. Marcel Dekker, New York, 2003. 